\title{
Algebraic Reconstruction of Current Dipoles and Quadrupoles in Three-Dimensional Space
}

\author{
Takaaki Nara \\ Graduate School of Information Science and Technology, The University of Tokyo, 7-3-1 Hongo, Bunkyo, Tokyo 113-8656, Japan \\ Correspondence should be addressed to Takaaki Nara; nara@mce.uec.ac.jp
}

Received 27 July 2012; Accepted 28 December 2012

Academic Editor: Valery Yakhno

Copyright (c) 2013 Takaaki Nara. This is an open access article distributed under the Creative Commons Attribution License, which permits unrestricted use, distribution, and reproduction in any medium, provided the original work is properly cited.

\begin{abstract}
This paper presents an algebraic method for an inverse source problem for the Poisson equation where the source consists of dipoles and quadrupoles. This source model is significant in the magnetoencephalography inverse problem. The proposed method identifies the source parameters directly and algebraically using data without requiring an initial parameter estimate or iterative computation of the forward solution. The obtained parameters could be used for the initial solution in an optimization-based algorithm for further refinement.
\end{abstract}

\section{Introduction}

Inverse source problems for the Poisson equation have a wide variety of applications such as bioelectromagnetic inverse problems. Magnetoencephalography (MEG) and electroencephalography (EEG) are typical examples in which the current source inside the human brain is inversely reconstructed from measurements of the magnetic field outside the head and the electric potential on the head surface [1-3]. Due to the quasistatic property of the magnetic and electric fields, MEG and EEG exhibit high temporal resolution compared to other tools for the noninvasive inspection of brains. Hence, the development of a stable inverse algorithm which reconstructs the source with high spatial resolution is of importance for MEG and EEG.

Conventional algorithms for the MEG inverse problem, whose solution is generally nonunique [4], can be divided into two categories [2]: the parametric and imaging approaches. The parametric approach assumes that the current source inside the brain is expressed by a relatively small number of equivalent current dipoles (ECDs), which guarantees the uniqueness of the solution [4] and finds their number, positions, and dipole moments. A typical method uses optimization-based algorithms to minimize the squared sum of the difference between the forward solution and data measured at a finite number of sensor positions (see, e.g., [5]). The forward solution is given by either the Geselowitz formula, which assumes that the head consists of arbitrarliy-shaped layered domains, or the Sarvas formula, which assumes that the head consists of concentric spheres [1]. A problem with optimization-based algorithms is that they require an initial solution close to the true solution, or the algorithm will often converge to a local minimum. To resolve this problem, algebraic methods have been proposed and are gaining increased importance [6-10]. In these methods, the ECD parameters are reconstructed directly and algebraically from the boundary measurements of the magnetic field.

In contrast, the second category of inverse algorithms, the imaging approach, assumes that the many dipoles are set at the vertices of a mesh overlaying the cortical surface and solves for their dipole moments. An advantage of this approach is that the spatially distributed source can be reconstructed by solving linear equations for the unknown dipole moments. However, it has the disadvantages that the solution is not unique and an oversmoothed solution is often obtained by regularization, such as the minimum $L_{2}$ norm solution [11]. A method using the minimum $L_{1}$ norm solution has also been proposed to reconstruct a sparse source [12].

Recently, a new method, the multipolar representation of the source, has been developed that incorporates some of the advantages of the above two methods, and has attracted considerable attention [13-18]. In this model, instead of 
expressing the current source by an equivalent current dipole, an equivalent dipole and quadrupole $[15,16]$ or an equivalent dipole and octopole $[13,14]$ are used where the quadrupole or octopole is determined depending on the spatial extent of the support of the current source. Jerbi et al. showed that the centroid of the spatially distributed source, which they called a patch source, can be estimated more accurately using the dipole and quadrupole model than using the dipole model by means of the nonlinear least squares method [16]. We considered a two-dimensional (2D) problem using a complex analysis framework and proposed an algebraic method to reconstruct the dipole and quadrupole parameters directly from the boundary measurements of the meromorphic function [17]. The aim of the current paper is to extend our algebraic method from the $2 \mathrm{D}$ case to the $3 \mathrm{D}$ case so that the dipole and quadrupoles, which equivalently represent the neural current, can be reconstructed from the magnetic field data without an initial parameter estimate or iterative computing of the forward solution. In $[19,20]$, we proposed an algebraic method when the dipoles were distributed in a plane parallel to the $x y$-plane, which is a very special case and is severely restricted in its practical usage. This paper derives a method for the general case. From a practical viewpoint, our method can provide an estimate of the number of patches as well as a good initial solution close to the true solution for optimization-based algorithms.

This paper is organized as follows. In Section 2, the forward problem with the dipole-quadrupole source model is summarized and the inverse problem is formulated. In Section 3, we propose a method to reconstruct the $x y$ coordinates of the dipole-quadrupole source by solving simultaneous algebraic equations of second degree. A method to estimate the $z$-coordinates is also proposed. Section 4 is devoted to numerical simulations for estimating spatially distributed dipoles.

\section{Forward and Inverse Problems with the Dipole and Quadrupole Source Model}

Let $\Omega_{1}, \Omega_{2}, \Omega_{3}$, and $\Omega_{4}$ be concentric balls centered at the origin in $3 \mathrm{D}$ space, where $\Omega_{i} \subset \Omega_{i+1}$ for $i=1,2,3$, as shown in Figure 1. Here, $\Omega_{1}, \Omega_{2} / \Omega_{1}$, and $\Omega_{3} / \Omega_{2}$ represent the brain, skull, and scalp, respectively. $\Omega_{3}$ represents the head. We assume that the radial component of the magnetic field is measured on the sphere $\Gamma=\partial \Omega_{4}$ with the radius of $R$. Although we use this simple head model as well as the spherical sensor surface in this paper, our method can be extended to a more realistic case when the head is modeled by a piecewise homogeneous layered domain and the sensors are set on an arbitrarily shaped surface as assumed in the algebraic method for the dipole source model [10]. Let $\mathbf{J}_{p}(\mathbf{r})$ represent the neural currents in the innermost domain $\Omega_{1}$. The magnetic permeability is assumed to be constant $\mu_{0}$ in the whole space.

First, we derive the representation of the radial magnetic field in terms of the equivalent dipoles and quadrupoles, which has already been given in [15], to determine how the quadrupole terms equivalently express the spatial extent of the source.

It is known that with this head model, the radial component of the magnetic field at $\mathbf{r}$ is equal to that when $\mathbf{J}_{p}(\mathbf{r})$ exists in infinite homogeneous space and is given by the Biot-Savart law:

$$
\mathbf{r} \cdot \mathbf{B}(\mathbf{r})=\frac{\mu_{0}}{4 \pi} \int_{\Omega_{1}}\left(\nabla^{\prime} \frac{1}{\left|\mathbf{r}-\mathbf{r}^{\prime}\right|} \times \mathbf{r}\right) \cdot \mathbf{J}_{p}\left(\mathbf{r}^{\prime}\right) d v^{\prime} .
$$

Let us assume that the neural activity is localized at several domains $D_{k}\left(\subset \Omega_{1}\right)$ for $k=1,2, \ldots, N$ so that $\mathbf{J}_{p}(\mathbf{r})$ is given by

$$
\mathbf{J}_{p}(\mathbf{r})=\sum_{k=1}^{N} \mathbf{j}_{p}(\mathbf{r}) \chi_{D_{k}},
$$

where $\chi_{D_{k}}$ is the characteristic function. Then, the radial component of the magnetic field is given by

$$
\mathbf{r} \cdot \mathbf{B}(\mathbf{r})=\frac{\mu_{0}}{4 \pi} \sum_{k=1}^{N} \int_{D_{k}}\left(\nabla^{\prime} \frac{1}{\left|\mathbf{r}-\mathbf{r}^{\prime}\right|} \times \mathbf{r}\right) \cdot \mathbf{j}_{p}\left(\mathbf{r}^{\prime}\right) d v^{\prime} .
$$

Assume that $D_{k}$ is contained in a ball $B_{k} \subset \Omega_{1}$, and there exists a "centroid" $\mathbf{r}_{k}$ of $D_{k}$ such that

$$
\left|\mathbf{r}-\mathbf{r}_{k}\right|>\left|\mathbf{r}^{\prime}-\mathbf{r}_{k}\right|, \quad \forall \mathbf{r} \in \Gamma, \mathbf{r}^{\prime} \in D_{k}
$$

From the Taylor series expansion, we have

$$
\begin{aligned}
\nabla^{\prime} \frac{1}{\left|\mathbf{r}-\mathbf{r}^{\prime}\right|}= & \left.\nabla^{\prime} \frac{1}{\left|\mathbf{r}-\mathbf{r}^{\prime}\right|}\right|_{\mathbf{r}^{\prime}=\mathbf{r}_{k}} \\
& +\left.\nabla^{\prime} \nabla^{\prime} \frac{1}{\left|\mathbf{r}-\mathbf{r}^{\prime}\right|}\right|_{\mathbf{r}^{\prime}=\mathbf{r}_{k}}\left(\mathbf{r}^{\prime}-\mathbf{r}_{k}\right)+\cdots
\end{aligned}
$$

Substituting this expansion into the right-hand side of (3) gives

$$
\begin{aligned}
\mathbf{r} \cdot \mathbf{B}(\mathbf{r})= & \frac{\mu_{0}}{4 \pi} \sum_{k=1}^{N} \frac{\mathbf{r}-\mathbf{r}_{k}}{\left|\mathbf{r}-\mathbf{r}_{k}\right|^{3}} \cdot\left(\mathbf{r}_{k} \times \int_{D_{k}} \mathbf{j}_{p}\left(\mathbf{r}^{\prime}\right) d v^{\prime}\right) \\
& +\frac{\mu_{0}}{4 \pi} \sum_{k=1}^{N} \frac{3\left(\mathbf{r} \times \mathbf{r}_{k}\right)\left(\mathbf{r}-\mathbf{r}_{k}\right)+\left|\mathbf{r}-\mathbf{r}_{k}\right|^{2} X_{r}}{\left|\mathbf{r}-\mathbf{r}_{k}\right|^{5}} \\
: & \int_{D_{k}} \mathbf{j}_{p}\left(\mathbf{r}^{\prime}\right)\left(\mathbf{r}^{\prime}-\mathbf{r}_{k}\right) d v^{\prime}+\cdots,
\end{aligned}
$$

where $X_{r}$ is the cross-product tensor defined by

$$
X_{r} \mathbf{a}=\mathbf{r} \times \mathbf{a},
$$

and hence is written as

$$
X_{r}=\left(\begin{array}{ccc}
0 & -z & y \\
z & 0 & -x \\
-y & x & 0
\end{array}\right) \quad \text { for } \mathbf{r}=(x, y, z)^{T}
$$

In (6), “:” represents the tensor contraction defined by $A$ : $B=\sum_{i=1}^{3} \sum_{j=1}^{3} A_{i j} B_{i j}$ for second-order tensors $A$ and $B$ whose 
$(i, j)$ components are given by $A_{i j}$ and $B_{i j}$, respectively. See Appendix A for the derivation of (6).

Now, let

$$
\begin{gathered}
\mathbf{p}_{k} \equiv \int_{D_{k}} \mathbf{j}_{p}\left(\mathbf{r}^{\prime}\right) d v^{\prime} \\
Q_{k} \equiv \frac{1}{2}\left(\int_{D_{k}} \mathbf{j}_{p}\left(\mathbf{r}^{\prime}\right)\left(\mathbf{r}^{\prime}-\mathbf{r}_{k}\right)+\left(\mathbf{r}^{\prime}-\mathbf{r}_{k}\right) \mathbf{j}_{p}\left(\mathbf{r}^{\prime}\right)\right) d v^{\prime} \\
=\left(\begin{array}{lll}
q_{x x, k} & q_{x y, k} & q_{x z, k} \\
q_{y x, k} & q_{y y, k} & q_{y z, k} \\
q_{z x, k} & q_{z y, k} & q_{z z, k}
\end{array}\right),
\end{gathered}
$$

then we have

$$
\begin{aligned}
\mathbf{r} \cdot \mathbf{B}(\mathbf{r}) & =\frac{\mu_{0}}{4 \pi} \sum_{k=1}^{N}\left(\mathbf{r}_{k} \times \mathbf{p}_{k}\right) \cdot \frac{\mathbf{r}-\mathbf{r}_{k}}{\left|\mathbf{r}-\mathbf{r}_{k}\right|^{3}}+\frac{\mu_{0}}{4 \pi} \sum_{k=1}^{N} Q_{k} \\
& : \frac{3\left(\mathbf{r} \times \mathbf{r}_{k}\right)\left(\mathbf{r}-\mathbf{r}_{k}\right)+\left|\mathbf{r}-\mathbf{r}_{k}\right|^{2} X_{r}}{\left|\mathbf{r}-\mathbf{r}_{k}\right|^{5}}+\cdots .
\end{aligned}
$$

Note here that we define $Q_{k}$ to be symmetric, $q_{i j, k}=q_{j i, k}$, since in (6) only the symmetric part of $\int_{D_{k}} \mathbf{j}_{p}\left(\mathbf{r}^{\prime}\right)\left(\mathbf{r}^{\prime}-\mathbf{r}_{k}\right) d v^{\prime}$ contributes to $\mathbf{r} \cdot \mathbf{B}$ as shown in [15]. Equation (10) was given in (46) in [15] (when $N=1$ ). The first term in (10) is the magnetic field created by an "equivalent current dipole" $\mathbf{p}_{k}$, and the second term is the magnetic field created by an "equivalent current quadrupole" $Q_{k}$. Note here that $\mathbf{p}_{k}$ does not depend on the size of $D_{k}$, while $Q_{k}$ depends on the spatial extent of $\mathbf{j}_{p}$ in $D_{k} . Q_{k}$ is a $3 \times 3$ tensor of order 2 and is called the quadrupole moment tensor.

Here, we can show that the truncation of (10) up to the second term,

$$
\begin{aligned}
\mathbf{r} \cdot \mathbf{B}(\mathbf{r})=\frac{\mu_{0}}{4 \pi} \sum_{k=1}^{N}( & \frac{\left(\mathbf{r}_{k} \times \mathbf{p}_{k}\right) \cdot\left(\mathbf{r}-\mathbf{r}_{k}\right)}{\left|\mathbf{r}-\mathbf{r}_{k}\right|^{3}} \\
& \left.+Q_{k}: \frac{3\left(\mathbf{r} \times \mathbf{r}_{k}\right)\left(\mathbf{r}-\mathbf{r}_{k}\right)+\left|\mathbf{r}-\mathbf{r}_{k}\right|^{2} X_{r}}{\left|\mathbf{r}-\mathbf{r}_{k}\right|^{5}}\right),
\end{aligned}
$$

is the forward solution for the source model given by

$$
\mathbf{J}_{p}(\mathbf{r})=\sum_{k=1}^{N} \mathbf{p}_{k} \delta\left(\mathbf{r}-\mathbf{r}_{k}\right)+\sum_{k=1}^{N} Q_{k} \nabla \delta\left(\mathbf{r}-\mathbf{r}_{k}\right),
$$

where $\delta$ is the Dirac delta function (see Appendix B). We call (12) the "equivalent dipole and quadrupole source model" or simply the "dipole-quadrupole source model." We are now ready to state our inverse problem.

Problem 1. Assume that a head $\Omega_{3}$ is modeled by a spherically layered domain. Assume that the neural current is expressed by (12) supported in the inner-most ball $\Omega_{1}$ representing the brain. Then, reconstruct the number $N$ and positions $\mathbf{r}_{k}$ of the dipole-quadrupole source in (12) from the radial component of the magnetic field measured on the spherical boundary $\Gamma$ which encloses $\Omega_{3}$.
Remark 1. Once the number and positions of the dipolequadrupole source are determined, the dipole moments $\mathbf{p}_{k}$ and quadrupole moment tensors $Q_{k}$ can be obtained by solving the relevant linear equation (11). In this paper, we concentrate on reconstructing $N$ and $\mathbf{r}_{k}$.

\section{Algebraic Reconstruction Method}

According to (88) in [15], we can express (1) by the multipole expansion

$$
\mathbf{r} \cdot \mathbf{B}(\mathbf{r})=\mu_{0} \sum_{l=0}^{\infty} \sum_{m=-l}^{l} \frac{l+1}{2 l+1} M_{l m} \frac{\widehat{Y}_{l m}^{*}(\theta, \phi)}{r^{l+1}}
$$

where

$$
\begin{aligned}
\widehat{Y}_{l m}(\theta, \phi)= & \sqrt{\frac{2 l+1}{4 \pi} \frac{(l-m) !}{(l+m) !}} \\
& \times Y_{l m}(\theta, \phi): \text { normalized spherical harmonics, } \\
Y_{l m}(\theta, \phi) & =P_{l}^{m}(\cos \theta) \mathrm{e}^{i m \phi}: \text { spherical harmonics, }
\end{aligned}
$$

and $P_{l}^{m}(\cos \theta)$ are the associated Legendre polynomials. As shown in (84) in [15], the multipole moment has the following relationship with $\mathbf{J}_{p}$ :

$$
M_{l m}=\frac{1}{l+1} \int_{\Omega_{1}}\left[\nabla^{\prime} r^{\prime l} \widehat{Y}_{l m}\left(\theta^{\prime}, \phi^{\prime}\right)\right] \cdot\left(\mathbf{r}^{\prime} \times \mathbf{J}_{p}\left(\mathbf{r}^{\prime}\right)\right) d^{3} r^{\prime} .
$$

Substituting (12) into (15) and using (B.1) gives

$$
\begin{aligned}
M_{l m}=\frac{1}{l+1} \sum_{k=1}^{N}\left(\left.\nabla^{\prime} r^{\prime l} \widehat{Y}_{l m}\left(\theta^{\prime}, \phi^{\prime}\right)\right|_{\mathbf{r}^{\prime}=\mathbf{r}_{k}}+Q_{k}\right. \\
\left.\quad:\left.\left(\nabla^{\prime}\left(\nabla^{\prime} r^{\prime l} \widehat{Y}_{l m}\left(\theta^{\prime}, \phi^{\prime}\right) \times \mathbf{r}^{\prime}\right)\right)^{T}\right|_{\mathbf{r}^{\prime}=\mathbf{r}_{k}}\right) .
\end{aligned}
$$

On the other hand, using the orthogonality of the spherical harmonics, the multipole moment is shown to have another relationship with the radial magnetic field on a spherical surface $\Gamma[21]$ :

$$
M_{l m}=\frac{2 l+1}{\mu_{0}(l+1)} \int_{\Gamma} \mathbf{n}^{\prime} \cdot \mathbf{B}\left(\mathbf{r}^{\prime}\right) R^{l} \widehat{Y}_{l m}\left(\theta^{\prime}, \phi^{\prime}\right) d S^{\prime},
$$

where $\mathbf{n}^{\prime}$ is the outward unit normal vector at $\mathbf{r}^{\prime}$ on $\Gamma$. Equating (16) and (17) for $l \geq m \geq 0$ gives algebraic equations relating the unknown parameters of the sources to the radial magnetic field on $\Gamma$. Although (17) requires the radial components of the magnetic field on the whole boundary $\Gamma$, Taulu et al. proposed a new approach for obtaining $M_{l m}$ from the data on the part of $\Gamma$ by using the truncated spherical harmonic expansion [22]. Using the method, we have algebraic equations about the unknown source parameters with the practically measurable MEG data. 
3.1. Algebraic Reconstruction of the $x$ - and $y$-Coordinates. As we derived the algebraic method for the dipole source model in [10], we now use the multipole moments of the sectorial harmonics represented by $l=m=n$, where $n \in \mathbb{N}$. From the relationship $r^{n} Y_{n n}(\theta, \phi)=(2 n-1) ! !(x+i y)^{n}$ [23], we have

$$
\begin{aligned}
\sum_{k=1}^{N}( & \left.\nabla(x+i y)^{n}\right|_{\mathbf{r}=\mathbf{r}_{k}} \cdot\left(\mathbf{r}_{k} \times \mathbf{p}_{k}\right) \\
& \left.+Q_{k}:\left.\left(\nabla\left(\nabla(x+i y)^{n} \times \mathbf{r}\right)\right)^{T}\right|_{\mathbf{r}=\mathbf{r}_{k}}\right) \\
= & \frac{2 n+1}{\mu_{0}} \int_{\Gamma} \mathbf{n} \cdot \mathbf{B}(\mathbf{r})(x+i y)^{n} d S .
\end{aligned}
$$

The primes in both sides are removed for simplicity. Since it holds that

$$
\begin{gathered}
\nabla(x+i y)^{n}=n\left(\begin{array}{l}
1 \\
i \\
0
\end{array}\right)(x+i y)^{n-1}, \\
\left(\nabla\left(\nabla(x+i y)^{n} \times \mathbf{r}\right)\right)^{T} \\
=n\left(\begin{array}{ccc}
i(n-1) z(x+i y)^{n-2} & -(n-1) z(x+i y)^{n-2} & i(x+i y)^{n-1} \\
-(n-1) z(x+i y)^{n-2} & -i(n-1) z(x+i y)^{n-2} & -(x+i y)^{n-1} \\
-i n(x+i y)^{n-1} & n(x+i y)^{n-1} & 0
\end{array}\right),
\end{gathered}
$$

we obtain

$$
\begin{gathered}
\left.\nabla(x+i y)\right|_{\mathbf{r}=\mathbf{r}_{k}} \cdot\left(\mathbf{r}_{k} \times \mathbf{p}_{k}\right)=n\left[\mathbf{r}_{k} \times \mathbf{p}_{k}\right]_{x+i y}\left(x_{k}+i y_{k}\right)^{n-1}, \\
Q_{k}:\left.\left(\nabla\left(\nabla(x+i y)^{n} \times \mathbf{r}\right)\right)^{T}\right|_{\mathbf{r}=\mathbf{r}_{k}} \\
=n(n-1)\left(\left(i\left(q_{x x, k}-q_{y y, k}\right)-2 q_{x y, k}\right) z_{k}\right. \\
\left.\quad+\left(q_{y z, k}-i q_{x z, k}\right)\left(x_{k}+i y_{k}\right)\right)\left(x_{k}+i y_{k}\right)^{n-2},
\end{gathered}
$$

where $\left[\mathbf{r}_{k} \times \mathbf{p}_{k}\right]_{x+i y}$ represents $\left(x\right.$-component of $\left.\mathbf{r}_{k} \times \mathbf{p}_{k}\right)+i \times$ $\left(y\right.$-component of $\mathbf{r}_{k} \times \mathbf{p}_{k}$ ). See Appendix $\mathrm{C}$ for the derivation of (21). Substituting (20) and (21) into (18) gives

$$
\begin{aligned}
\sum_{k=1}^{N}\left(\left[\mathbf{r}_{k} \times \mathbf{p}_{k}\right]_{x+i y}\left(x_{k}+i y_{k}\right)^{n-1}+(n-1)\right. \\
\times\left(\left(i\left(q_{x x, k}-q_{y y, k}\right)-2 q_{x y, k}\right) z_{k}\right. \\
\left.\left.\quad+\left(q_{y z, k}-i q_{x z, k}\right)\left(x_{k}+i y_{k}\right)\right)\left(x_{k}+i y_{k}\right)^{n-2}\right) \\
=\frac{2 n+1}{\mu_{0} n} \int_{\Gamma} \mathbf{n} \cdot \mathbf{B}(x+i y)^{n} d S .
\end{aligned}
$$

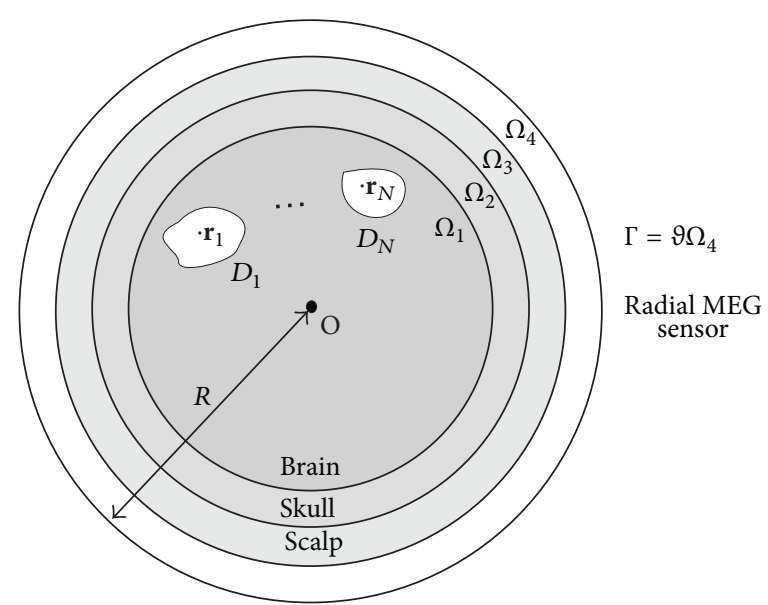

Figure 1: Head and sensor model.

We now put

$$
\begin{gathered}
\zeta_{k}=x_{k}+i y_{k}, \\
\mu_{k}=\left[\mathbf{r}_{k} \times \mathbf{p}_{k}\right]_{x+i y}, \\
v_{k}=\left(i\left(q_{x x, k}-q_{y y, k}\right)-2 q_{x y, k}\right) z_{k} \\
+\left(q_{y z, k}-i q_{x z, k}\right)\left(x_{k}+i y_{k}\right), \\
c_{n}=\frac{2 n+3}{\mu_{0}(n+1)} \int_{\Gamma} \mathbf{n} \cdot \mathbf{B}(x+i y)^{n+1} d S
\end{gathered}
$$

and rewrite (22) as

$$
\sum_{k=1}^{N} \mu_{k} \zeta_{k}^{n}+n \sum_{k=1}^{N} v_{k} \zeta_{k}^{n-1}=c_{n} \quad\left(n \in \mathbb{N}^{*}\right)
$$

Comparing with the special case where the dipoles were distributed in a plane parallel to the $x y$-plane in [20], one finds that $v_{k}$ has an extra term: $\left(q_{y z, k}-i q_{x z, k}\right)\left(x_{k}+i y_{k}\right)$.

Equation (24) has the same form as that of (6) in [17], and consequently the dipole-quadrupole position projected on the $x y$-plane, $\zeta_{k}(k=1,2, \ldots, N)$, can be algebraically reconstructed from $c_{n}(n=0,1, \ldots, 2 N-1)$, which is shown as follows. In Section 3.1, we assume that $N$ is known a priori and construct an algorithm to estimate $\zeta_{k}$ in (24). A method to estimate $N$ as well as $\mu_{k}$ and $\nu_{k}$ is then described in Section 3.2.

Let us define $\sigma_{1}, \sigma_{2}, \ldots, \sigma_{N}$ by the coefficients of

$$
\prod_{k=1}^{N}\left(\zeta-\zeta_{k}\right)=\zeta^{N}+\sigma_{1} \zeta^{N-1}+\cdots+\sigma_{N}
$$

It was proved in [17] that (24) can be transformed into the second-degree equations for $\sigma_{1}, \sigma_{2}, \ldots, \sigma_{N}$ :

$$
\boldsymbol{\sigma}^{T} H_{N, m} \boldsymbol{\sigma}=0 \quad\left(m \in \mathbb{N}^{*}\right),
$$


where $\boldsymbol{\sigma}=\left(\sigma_{N}, \ldots, \sigma_{1}, 1\right)^{T}$ and $H_{N, m}$ is the $(N+1) \times(N+1)$ Hankel matrix given by

$$
H_{N, m}=\left(\begin{array}{cccc}
c_{m} & c_{m+1} & \cdots & c_{m+N} \\
c_{m+1} & c_{m+2} & \cdots & c_{m+N+1} \\
\vdots & \vdots & \vdots & \vdots \\
c_{m+N} & c_{m+N+1} & \cdots & c_{m+2 N}
\end{array}\right) .
$$

Moreover, it has been shown that the $2 \mathrm{~N}$ equations (26) for $m=0,1, \ldots, 2 N-1$ can be turned into linear equations as follows. First, from those $2 N$ equations, we make $2 N-1$ equations

$$
\boldsymbol{\sigma}^{T} H_{N, m}^{(1)} \boldsymbol{\sigma}=0 \quad(m=0,1, \ldots, 2 N-2),
$$

where

$$
H_{N, m}^{(1)} \equiv c_{m+1} H_{N, m}-c_{m} H_{N, m+1} .
$$

By this definition, $H_{N, m}^{(1)}$ are the Hankel matrices whose $(1,1)$ components are zero. We denote their components by

$$
H_{N, m}^{(1)}=\left(\begin{array}{cccc}
0 & c_{m+1}^{(1)} & \cdots & c_{m+N}^{(1)} \\
c_{m+1}^{(1)} & c_{m+2}^{(1)} & \cdots & c_{m+N+1}^{(1)} \\
\vdots & \vdots & \vdots & \vdots \\
c_{m+N}^{(1)} & c_{m+N+1}^{(1)} & \cdots & c_{m+2 N}^{(1)}
\end{array}\right)
$$

Next, from those $2 N-1$ equations, we make $2 N-2$ equations:

$$
\boldsymbol{\sigma}^{T} H_{N, m}^{(2)} \boldsymbol{\sigma}=0 \quad(m=0,1, \ldots, 2 N-3),
$$

where

$$
H_{N, m}^{(2)}=c_{m+2}^{(1)} H_{N, m}^{(1)}-c_{m+1}^{(1)} H_{N, m+1}^{(1)} .
$$

By definition, $H_{N, m}^{(2)}$ are the Hankel matrices expressed by

$$
H_{N, m}^{(2)}=\left(\begin{array}{ccccc}
0 & 0 & c_{m+2}^{(2)} & \cdots & c_{m+N}^{(2)} \\
0 & c_{m+2}^{(2)} & \cdots & \cdots & c_{m+N+1}^{(2)} \\
c_{m+2}^{(2)} & \cdots & \cdots & \cdots & c_{m+N+1}^{(2)} \\
\vdots & \vdots & \vdots & \vdots & \vdots \\
c_{m+N}^{(2)} & c_{m+N+1}^{(2)} & \cdots & \cdots & c_{m+2 N}^{(2)}
\end{array}\right) .
$$

Note that the components along the first and second lines in the antidiagonal direction become zero. Repeating this procedure to eliminate the components on the $j$ th line in the anti-diagonal direction for $j=3, \ldots, 2 N-1$, we obtain

$$
\boldsymbol{\sigma}^{T} H_{N, m}^{(j)} \boldsymbol{\sigma}=0 \quad(m=0,1, \ldots, 2 N-1-j),
$$

where $H_{N, m}^{(j)}$ are the Hankel matrices whose components along the first through $j$ th lines in the anti-diagonal direction are zero. Here, (34) for $j=2 N-1$ is a linear equation for $\sigma_{N}$. Solving it and substituting the solution into (34) for $j=2 N-2$ gives a linear equation for $\sigma_{N-1}$. Repeating the backward substitution gives $\sigma_{N-2}, \ldots, \sigma_{1}$, successively. The conditions for the unique solvability of these linear equations are $\zeta_{i} \neq \zeta_{j}$ for $i \neq j$ and $v_{k} \neq 0$. See [17] for more details. Once $\sigma_{1}, \ldots, \sigma_{N}$ are obtained, we have $\zeta_{k}$ as the roots of $\zeta^{N}+\sigma_{1} \zeta^{N-1}+\cdots+\sigma_{N}=$ 0 .

In a practical situation when data includes noise, the above procedure proposed in [17] to transform the seconddegree equations (26) into linear equations (34) to obtain $\sigma_{N}, \ldots, \sigma_{1}$ may be sensitive to the noise due to the cancellation. To reduce the cancellation error, we propose the following algorithm. First, we solve the second-degree equations (26) for $m=0, \ldots, N-1$ using a well-established method for solving simultaneous algebraic equations, for example, by means of the Gröbner bases. Then, out of the obtained $2^{N}$ solutions, $\left(\sigma_{1, j}, \ldots, \sigma_{N, j}\right)$ for $j=1, \ldots, 2^{N}$, we choose one which minimizes the sum of the absolute values of the lefthand sides of (26) for $m=N, \ldots, 2 N-1$ given by

$$
\sum_{m=N}^{2 N-1}\left|\boldsymbol{\sigma}^{T} H_{N, m} \boldsymbol{\sigma}\right| \text {. }
$$

In other words, to obtain the theoretically unique solution of (26) for $m=0, \ldots, 2 N-1$, first we find $2^{N}$ candidates from (26) for $m=0, \ldots, N-1$ and then choose the one which best explains the remaining equations (26) for $m=N, \ldots, 2 N-1$.

3.2. Reconstruction of $\mu_{k}, v_{k}$, and $N$. To estimate $N$, following the method for the dipole source model [10], we assume that there are $N^{\prime}(>N)$ dipole-quadrupole sources and then estimate $\zeta_{k}$ for $k=1,2, \ldots, N^{\prime}$ using the algorithm in Section 3.1. Once they are obtained, $\mu_{k}$ and $v_{k}$ for $k=1,2, \ldots, N^{\prime}$ are linearly solved using (24) for $n=0,1, \ldots, 2 N^{\prime}-1$. Then, we compute $\left|\mu_{n+1} / \mu_{n}\right|$ and $\left|v_{n+1} / v_{n}\right|$ for $n=1,2, \ldots, N^{\prime}-1$, which are expected to be sufficiently small when $n=N$. Practically, we estimate $N$ such that these ratios become smaller than some threshold set a priori. The thresholds should be determined by the ratios of the noise level contained in the data to the dipole and quadrupole strength which can be regarded as a physiologically meaningful source. As for the dipole source model in the $2 \mathrm{D}$ problem, the threshold is theoretically evaluated in the context of the Padé approximation [24]. A similar theory for the dipole-quadrupole source model, although greatly required, is left for further research; in this paper we show only numerical simulations in Section 4 .

3.3. Algebraic Reconstruction of the $z$-Coordinates. To determine the $z$-coordinates of the dipole-quadrupole positions, after we determine the $x y$-coordinates of the source, we use the method proposed in [7]. We put $l=n+1$ and $m=n$ in (16) and (17), where $n \in \mathbb{N}^{*}$. From the identity $r^{n+1} \widehat{Y}_{n+1, n}=$ $(2 n+1) !(x+i y)^{n} z[23]$, we have

$$
\begin{aligned}
& \sum_{k=1}^{N}(\left.(x+i y)^{n} z\right|_{\mathbf{r}=\mathbf{r}_{k}} \cdot\left(\mathbf{r}_{k} \times \mathbf{p}_{k}\right) \\
&\left.+Q_{k}:\left.\left(\nabla\left(\nabla(x+i y)^{n} z \times \mathbf{r}\right)\right)^{T}\right|_{\mathbf{r}=\mathbf{r}_{k}}\right) \\
&=\frac{2 n+3}{\mu_{0}} \int_{\Gamma} \mathbf{n} \cdot \mathbf{B}(\mathbf{r})(x+i y)^{n} z d S .
\end{aligned}
$$


Now, let

$$
\phi_{j}(t) \equiv\left(t-\zeta_{j}\right)^{2} \prod_{l=1, l \neq j}^{N}\left(t-\zeta_{l}\right)^{3} \equiv \sum_{n=0}^{3 N-1} \alpha_{n} t^{n} .
$$

Then, from $\sum_{n=0}^{3 N-1} \alpha_{n} \times(18)$ and $\sum_{n=0}^{3 N-1} \alpha_{n} \times(36)$, we obtain

$$
\begin{gathered}
\sum_{k=1}^{N}\left(\left.\nabla \phi_{j}(x+i y)\right|_{\mathbf{r}=\mathbf{r}_{k}} \cdot\left(\mathbf{r}_{k} \times \mathbf{p}_{k}\right)\right. \\
\left.+Q_{k}:\left.\left(\nabla\left(\nabla \phi_{j}(x+i y) \times \mathbf{r}\right)\right)^{T}\right|_{\mathbf{r}=\mathbf{r}_{k}}\right) \\
=\frac{1}{\mu_{0}} \sum_{n=0}^{3 N-1}(2 n+1) \alpha_{n} \int_{\Gamma} \mathbf{n} \cdot \mathbf{B}(\mathbf{r})(x+i y)^{n} d S \\
\sum_{k=1}^{N}\left(\left.\nabla \phi_{j}(x+i y) z\right|_{\mathbf{r}=\mathbf{r}_{k}} \cdot\left(\mathbf{r}_{k} \times \mathbf{p}_{k}\right)\right. \\
\left.+Q_{k}:\left.\left(\nabla\left(\nabla \phi_{j}(x+i y) z \times \mathbf{r}\right)\right)^{T}\right|_{\mathbf{r}=\mathbf{r}_{k}}\right) \\
=\frac{1}{\mu_{0}} \sum_{n=0}^{3 N-1}(2 n+3) \alpha_{n} \int_{\Gamma} \mathbf{n} \cdot \mathbf{B}(\mathbf{r})(x+i y)^{n} z d S,
\end{gathered}
$$

respectively. In the left-hand sides of (38) and (39),

$$
\begin{gathered}
\left.\nabla \phi_{j}(x+i y)\right|_{\mathbf{r}=\mathbf{r}_{k}}=\left(\begin{array}{c}
\phi_{j}^{\prime}\left(\zeta_{k}\right) \\
i \phi_{j}^{\prime}\left(\zeta_{k}\right) \\
0
\end{array}\right)=\mathbf{0}, \\
\left.\nabla \phi_{j}(x+i y) z\right|_{\mathbf{r}=\mathbf{r}_{k}}=\left(\begin{array}{c}
z \phi_{j}^{\prime}\left(\zeta_{k}\right) \\
i z \phi_{j}^{\prime}\left(\zeta_{k}\right) \\
\phi_{j}\left(\zeta_{k}\right)
\end{array}\right)=\mathbf{0}
\end{gathered}
$$

since from (37) it holds that $\phi_{j}\left(\zeta_{k}\right)=\phi_{j}^{\prime}\left(\zeta_{k}\right)=0$ for all $k=$ $1,2, \ldots, N$ irrespective of whether or not $k$ is equal to $j$. Also, we have

$$
\begin{gathered}
\left.\nabla\left(\nabla \phi_{j}(x+i y) \times \mathbf{r}\right)\right|_{\mathbf{r}=\mathbf{r}_{k}}=\delta_{j k} \Phi_{j}, \\
\left.\nabla\left(\nabla \phi_{j}(x+i y) z \times \mathbf{r}\right)\right|_{\mathbf{r}=\mathbf{r}_{k}}=z_{j} \delta_{j k} \Phi_{j},
\end{gathered}
$$

where $\delta_{j k}$ is the Kronecker delta, and

$$
\Phi_{j}=2 \prod_{l=1, l \neq j}^{N}\left(\zeta_{j}-\zeta_{l}\right)^{3}\left(\begin{array}{ccc}
i z_{j} & -z_{j} & -i \zeta_{j} \\
-z_{j} & -i z_{j} & \zeta_{j} \\
0 & 0 & 0
\end{array}\right) .
$$

See Appendix D for the derivation of (42) and (43). Substituting (40) through (43) into (38) and (39) gives

$$
\begin{gathered}
Q_{j}: \Phi_{j}^{T}=\frac{1}{\mu_{0}} \sum_{n=0}^{3 N-1}(2 n+1) \alpha_{n} \int_{\Gamma} \mathbf{n} \cdot \mathbf{B}(\mathbf{r})(x+i y)^{n} d S, \\
Q_{j}: z_{j} \Phi_{j}^{T}=\frac{1}{\mu_{0}} \sum_{n=0}^{3 N-1}(2 n+3) \alpha_{n} \int_{\Gamma} \mathbf{n} \cdot \mathbf{B}(\mathbf{r})(x+i y)^{n} z d S,
\end{gathered}
$$

from which we obtain

$$
z_{j}=\frac{\sum_{n=0}^{3 N-1}(2 n+3) \alpha_{n} \int_{\Gamma} \mathbf{n} \cdot \mathbf{B}(\mathbf{r})(x+i y)^{n} z d S}{\sum_{n=0}^{3 N-1}(2 n+1) \alpha_{n} \int_{\Gamma} \mathbf{n} \cdot \mathbf{B}(\mathbf{r})(x+i y)^{n} d S} .
$$

This is the reconstruction formula of the $z$-coordinates: once $\zeta_{k}$ for $k=1,2, \ldots, N$ are obtained, then $z_{j}$ are determined using (46) for each $j=1,2, \ldots, N$ with $\alpha_{n}$ defined by (37).

\subsection{Algorithm. Our algorithm is summarized as follows.}

Step 1 (estimate the number of the dipole-quadrupole sources). (1) Assume that there are $N^{\prime}(>N)$ dipole-quadrupole sources. Using the algorithm in Step 2(1)-(5) below, where $N$ is replaced by $N^{\prime}$, obtain $\mu_{k}$ and $\nu_{k}$ for $k=1,2$, $\ldots, N^{\prime}$.

(2) Compute $\left|\mu_{n+1} / \mu_{n}\right|$ and $\left|\nu_{n+1} / \nu_{n}\right|$ for $n=1,2, \ldots, N^{\prime}-$ 1. When $\left|\mu_{\widehat{N}+1} / \mu_{\widehat{N}}\right|<\epsilon_{\mu}$ and $\left|\nu_{\widehat{N}+1} / \nu_{\widehat{N}}\right|<\epsilon_{v}$ for the thresholds $\epsilon_{\mu}$ and $\epsilon_{v}$, which are appropriately set a priori, we estimate $N$ to be $\widehat{N}$.

Step 2 (estimate the position parameters of $N$ dipole-quadrupole sources). (1) Given $\mathbf{n} \cdot \mathbf{B}$ on $\Gamma$, compute $c_{n}$ in (23) for $n=0,1, \ldots, 4 N-1$ and construct the Hankel matrices (27) for $m=0,1, \ldots, 2 N-1$.

(2) Solve the simultaneous second-degree equation (26) for $m=0,1, \ldots, N-1$, using the Gröbner bases to get $2^{N}$ solutions.

(3) Out of the $2^{N}$ solutions, choose one solution $\sigma_{1}$, $\ldots, \sigma_{N}$ that minimizes (35).

(4) Solve $z^{N}+\sigma_{1} z^{N-1}+\cdots+\sigma_{N}=0$ to obtain $\zeta_{1}, \ldots, \zeta_{N}$.

(5) Solve (24) for $\mu_{k}$ and $v_{k}$, where $k=1, \ldots, N$.

(6) Using the obtained $\zeta_{1}, \ldots, \zeta_{N}$, compute $\alpha_{n}$ in (37) for $n=0,1, \ldots, 3 N-1$ and determine $z_{1}, \ldots, z_{N}$ using (46).

\section{Numerical Simulations}

In this section, our algorithm is verified numerically. $\Gamma$ was assumed to be a sphere with $R=0.12 \mathrm{~m}$ on which 361 measurement points were distributed uniformly using the spherical t-design [25]. To validate our algorithm for the dipolequadrupole model, we assumed that the data was available on the whole sphere which enclosed the source. Identification using data on a part of $\Gamma$ is an important aspect of future studies, for which the method proposed by Taulu et al. [22] would be useful.

4.1. Demonstration of Our Method. In this subsection, We examine the case where there are $N=2$ dipole-quadrupole sources whose parameters are given in Table 1. The theoretical data was computed using (11) to which Gaussian noise with $\sigma=10^{-2}$ was added where the noise level $\sigma$ is defined by the ratio of the standard deviation of the noise to the root mean squares of the data. 100 data sets with the different noise added were used for reconstruction.

First, to determine the number of the sources $N$, we assume that there are $N^{\prime}=3$ dipole-quadrupole sources. 


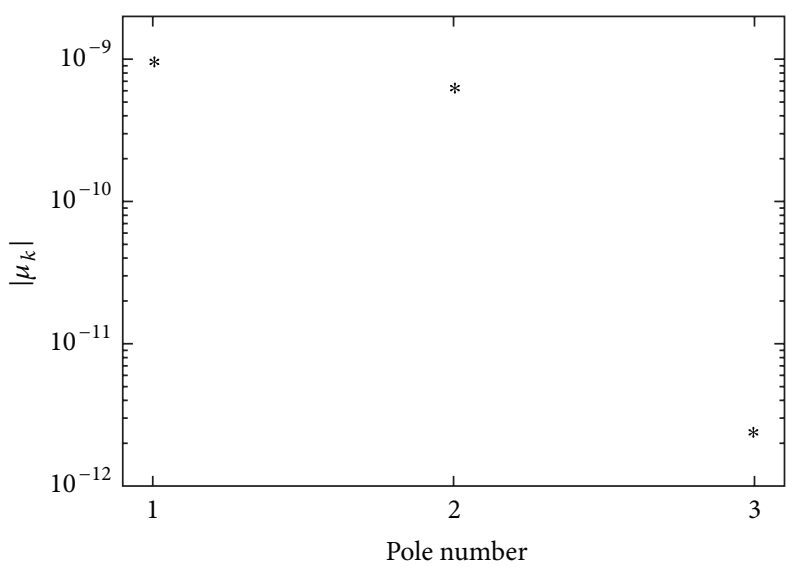

(a)

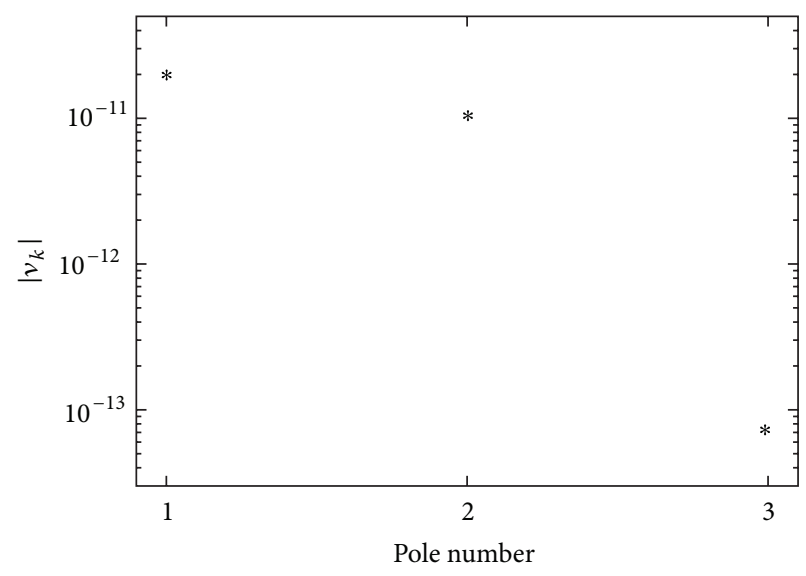

(b)

FIGURE 2: Estimation of $N$ : the obtained $\left|\mu_{k}\right|$ (a) and $\left|v_{k}\right|$ (b) for $k=1,2,3$ when assuming $N^{\prime}=3$. Since $\left|\mu_{3}\right|$ and $\left|v_{3}\right|$ are much smaller than $\left|\mu_{k}\right|$ and $\left|v_{k}\right|$ for $k=1,2$, respectively $\left(\left|\mu_{3} / \mu_{2}\right|=3.8 \times 10^{-3}\right.$ and $\left.\left|\nu_{3} / \nu_{2}\right|=7.2 \times 10^{-3}\right)$, we can judge that there are substantially $N=2$ dipole-quadrupole sources.

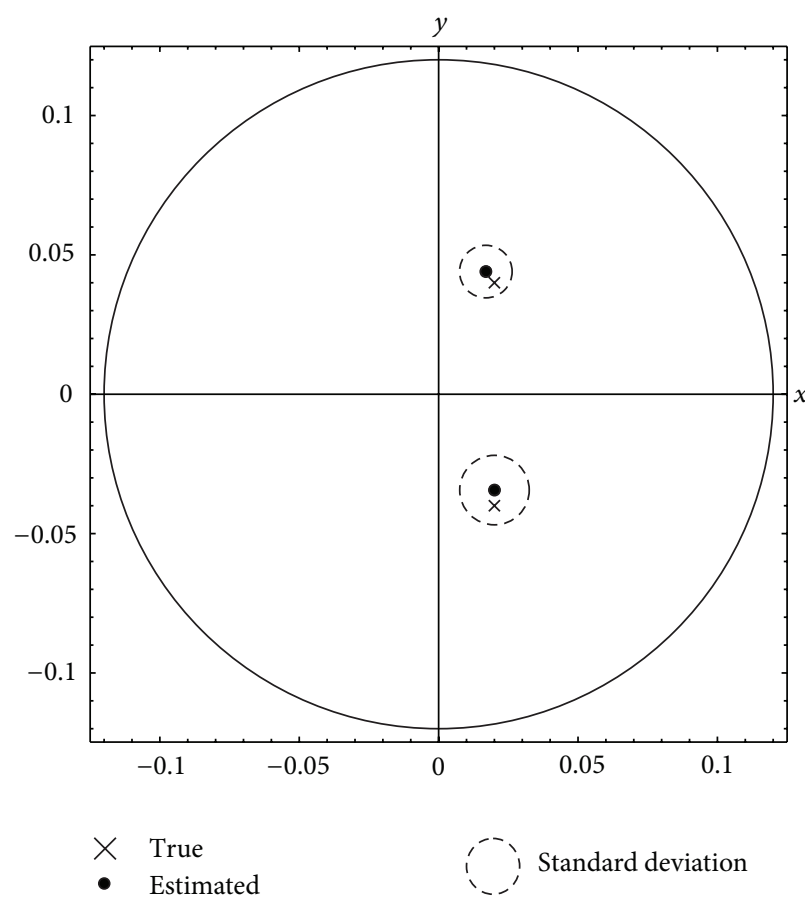

(a)

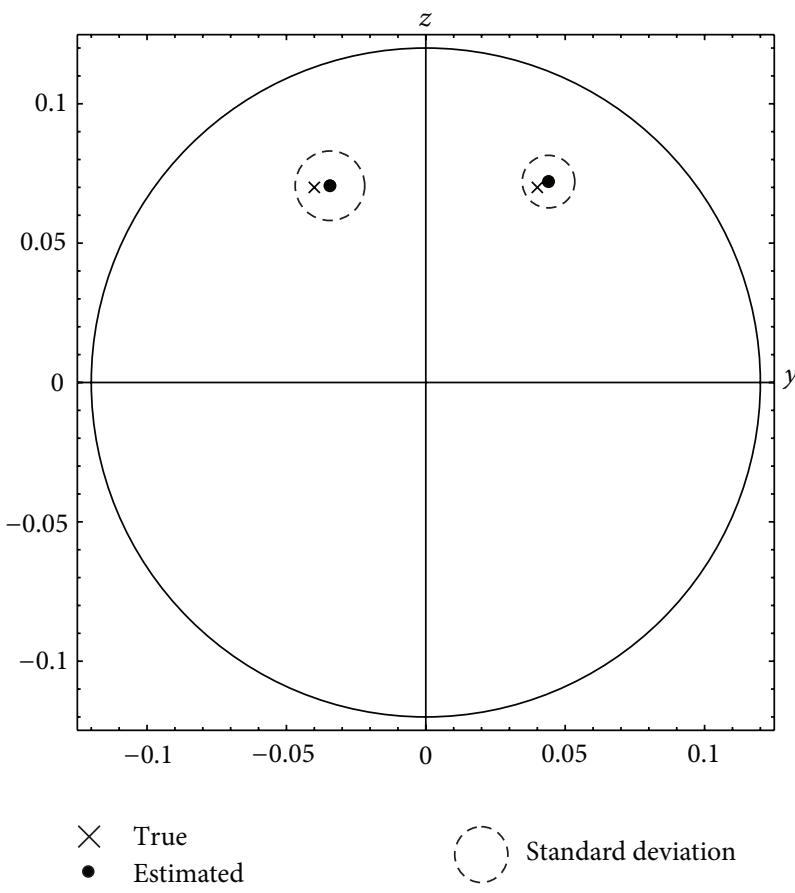

(b)

FIgURE 3: Localization result when $N=2$ where the noise level $\sigma=10^{-2}$.

Figure 2 shows the obtained $\left|\mu_{k}\right|$ and $\left|\nu_{k}\right|$ for $k=1,2,3$. One observes that $\left|\mu_{3}\right|$ and $\left|\nu_{3}\right|$ are much smaller than $\left|\mu_{k}\right|$ and $\left|v_{k}\right|$ for $k=1,2$, respectively. In fact, the geometric means of $\left|\mu_{3} / \mu_{2}\right|$ and $\left|\nu_{3} / \nu_{2}\right|$ for 100 data sets were $3.8 \times 10^{-3}$ and $7.2 \times 10^{-3}$, respectively. From this, we can judge that there are substantially $N=2$ poles.

Figure 3 shows the localization result projected on the $x y$ - and $y z$-planes. We find that, under this noise level, two dipole-quadrupole sources were stably reconstructed. The average and standard deviations of the computational time to identify the parameters of the two dipole-quadrupole sources were $0.69 \mathrm{sec}$ and $0.01 \mathrm{sec}$, respectively, using an Intel Core i7 CPU $870(2.93 \mathrm{GHz})$ with $8 \mathrm{~GB}$ RAM, showing that our algebraic algorithm can determine the source parameters with low computational cost.

4.2. Effect of Noise on Localization Accuracy. To examine the stability of our algorithm, the noise level $\sigma$ was varied in the wide range $10^{-8} \leq \sigma \leq 10^{-1}$. The source and sensor configuration were the same as in Section 4.1. Figure 4 shows 
TABLE 1: Dipole-quadrupole parameters when $N=2$.

\begin{tabular}{lccc}
\hline$k$ (pole no.) & $\mathbf{r}_{k}$ & $\mathbf{p}_{k}$ & $\left(q_{x x, k}, q_{x y, k}, q_{x z, k}, q_{y y, k}, q_{y z, k}, q_{z z, k}\right)$ \\
\hline 1 & $(0.02,0.04,0.07) \mathrm{m}$ & $(10,0,10) \mathrm{nAm}$ & $(1,1,0,2,1,0) \times 10^{-1} \mathrm{nAm}^{2}$ \\
2 & $(0.02,-0.04,0.07) \mathrm{m}$ & $(10,0,-10) \mathrm{nAm}$ & $(1,1,0,-2,1,0) \times 10^{-1} \mathrm{nAm}^{2}$ \\
\hline
\end{tabular}

TABLE 2: dipole-quadrupole parameters when $N=3$.

\begin{tabular}{lccc}
\hline$k$ (pole no.) & $\mathbf{r}_{k}$ & $\mathbf{p}_{k}$ & $\left(q_{x x, k}, q_{x y, k}, q_{x z, k}, q_{y y, k}, q_{y z, k}, q_{z z, k}\right)$ \\
\hline 1 & $(0.02,-0.04,0.07) \mathrm{mm}$ & $(10,0,10) \mathrm{nAm}$ & $(1,1,0,2,1,0) \times 10^{-1} \mathrm{nAm}^{2}$ \\
2 & $(-0.04,0,0.07) \mathrm{mm}$ & $(10,0,-10) \mathrm{nAm}$ & $(1,1,0,-2,1,0) \times 10^{-1} \mathrm{nAm}^{2}$ \\
3 & $(0,0.05,0.07) \mathrm{mm}$ & $(0,10,10) \mathrm{nAm}$ & $(2,1,0,1,1,0) \times 10^{-1} \mathrm{nAm}^{2}$ \\
\hline
\end{tabular}

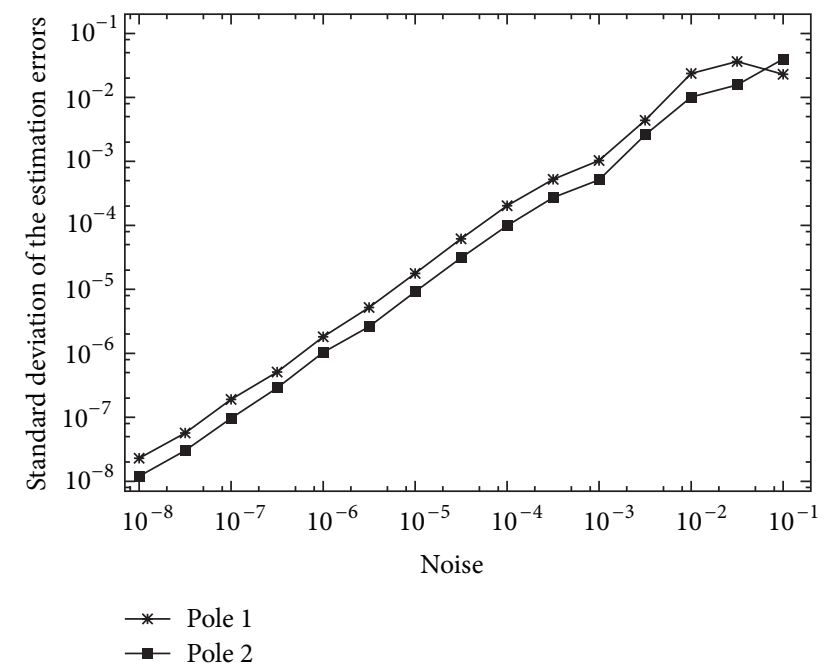

FIGURE 4: Standard deviation of the localization errors with respect to the noise level $\sigma$.

the standard deviation (s.d.) of the localization errors with respect to the noise level $\sigma$ for the two poles. It is observed that s.d. increases as $\sigma$ increases. For this source in the sphere with the radius $0.12 \mathrm{~m}$, to realize s.d. less than $0.01 \mathrm{~m}$, one finds that the noise level should be less than $\sigma=10^{-2}$.

\subsection{Effect of the Distance between Two Sources on Localization} Accuracy. We varied the distance between the two dipolequadrupole sources: the two dipole-quadrupole source were set at $(0.02, \pm d, 0.07)$ where $2 d$ was changed from 0.01 through 0.09 . Figure 5 shows the relative localization error (error divided by the radius of $\Gamma, 0.12$ ). It is observed that the distance between the two dipole-quadrupole sources should be larger than about 0.06 , that is the half of the radius of $\Gamma$, in order to obtain the relative error less than $10^{-2}$.

4.4. Example When $N=3$. We examine the case when there are $N=3$ dipole-quadrupole sources whose parameters are given in Table 2. The noise level was set at $\sigma=10^{-2}$. In this subsection, we assumed that $N$ is known a priori. Figure 6 shows the localization result. Comparing with the result when $N=2$ in Figure 3 , the biases as well as the standard deviations of the localization errors become

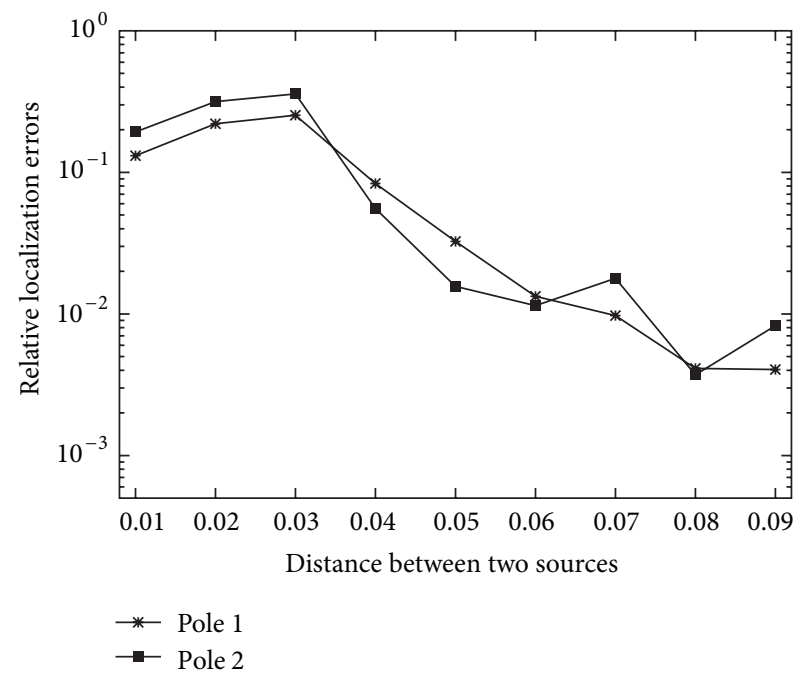

FIGURE 5: Relative localization error with respect to the distance between the two dipole-quadrupole sources.

larger when $N=3$. It is numerically shown in [26] that, when using dipole-only source model, stability gets worse when $N$ increases and it is difficult to estimate the dipoles greater than about $N=5$. The example in this subsection shows that it is more difficult to estimate the large number $(N \geq 3)$ of dipole-quadrupole sources. This is considered due to fast spatial decay of the quadrupolar field. However, the dipole-quadrupole source even with $N=1$ or 2 has an advantage that it can well estimate a spatially distributed sources, especially when they are modeled with two, close, oppositely directed dipoles, which is a weak point of the conventional dipole model. This is shown in the next subsection.

4.5. Identification of Dipoles Distributed on Curved Surfaces. In this subsection, we compare our algebraic method assuming the dipole-quadrupole model (DQM) with a conventional algebraic method assuming the dipole model (DM) for estimating the spatially distributed dipole sources. To model dipoles on cerebral convolutions, we assume that dipoles are placed on a mesh on a half-cylinder with a radius of $r=$ $5 \mathrm{~mm}$ and a height of $h=5 \mathrm{~mm}$, as shown in Figure 7. There are six dipoles in the circumferential direction by five in 


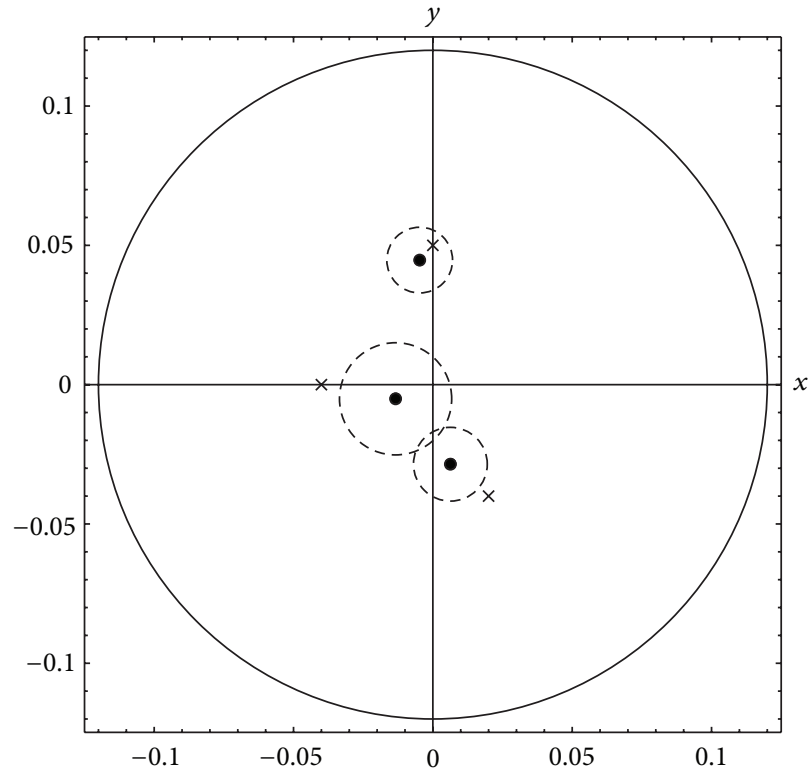

$\times$ True

- Estimated

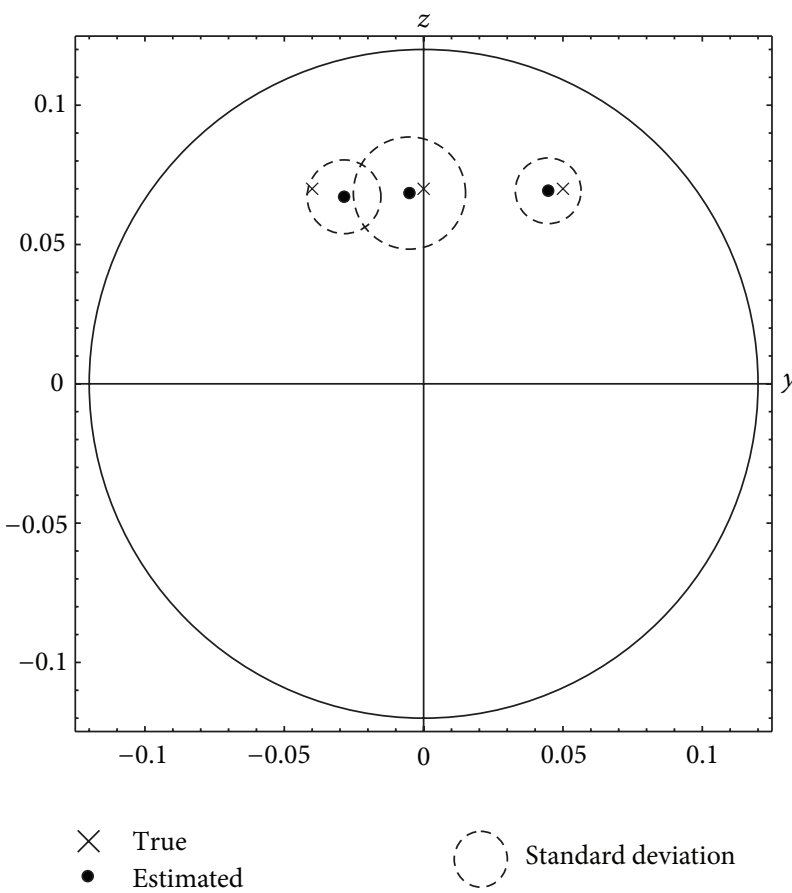

(b)

Figure 6: Localization result when $N=3$.

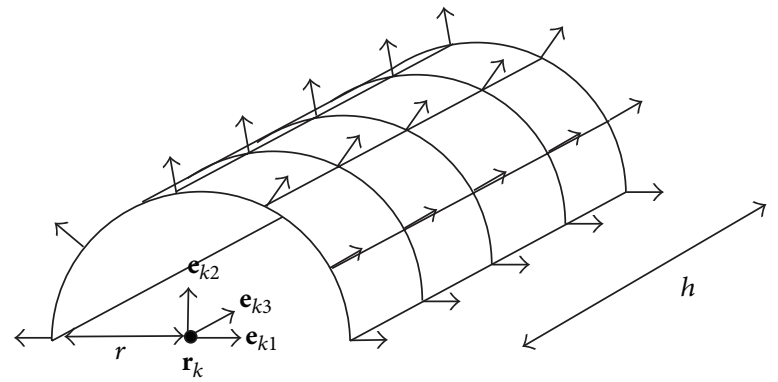

FIgURE 7: Source of distributed dipoles on a half-cylinder.

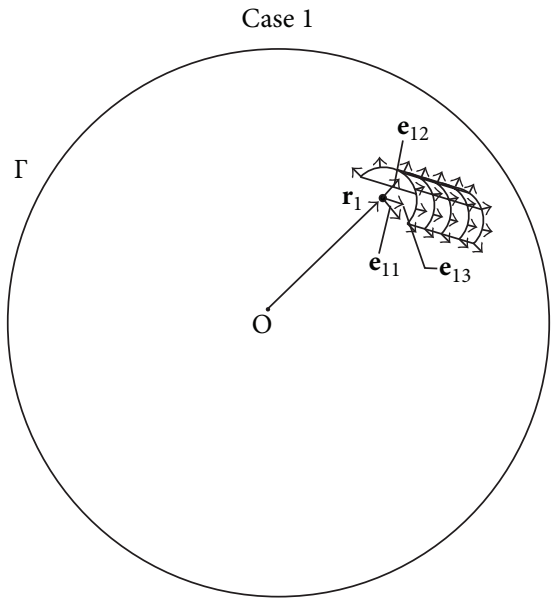

(a)

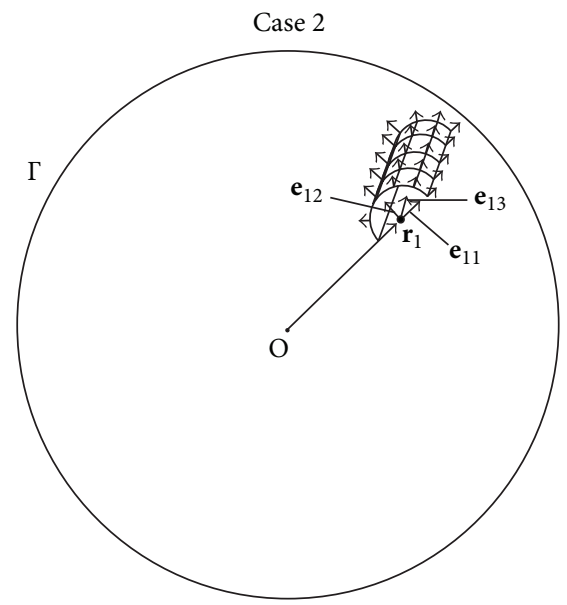

(b)

Figure 8: Cases 1 and 2 of the cylindrically distributed dipoles. 


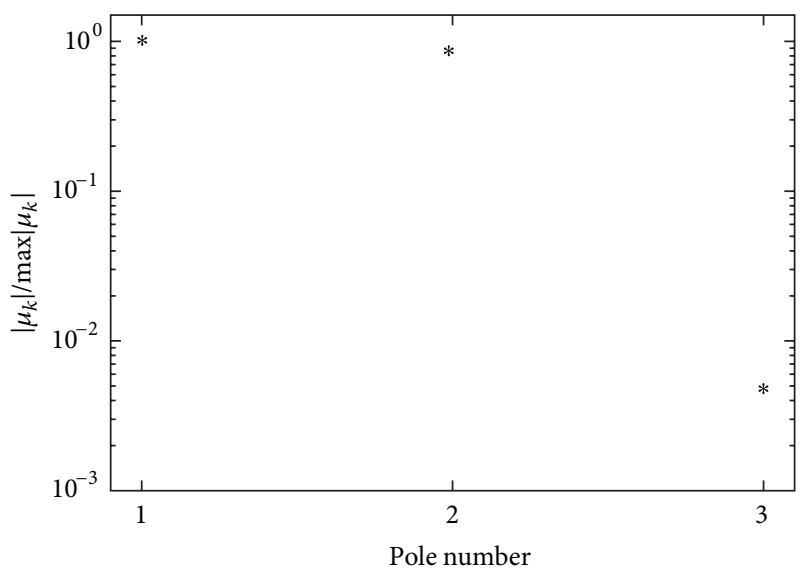

Figure 9: Estimation of $N$ in Case 1 using the dipole model (DM) when assuming $N^{\prime}=3$. From $\left|\mu_{3} / \mu_{2}\right|=0.005$, DM estimates $N=2$.

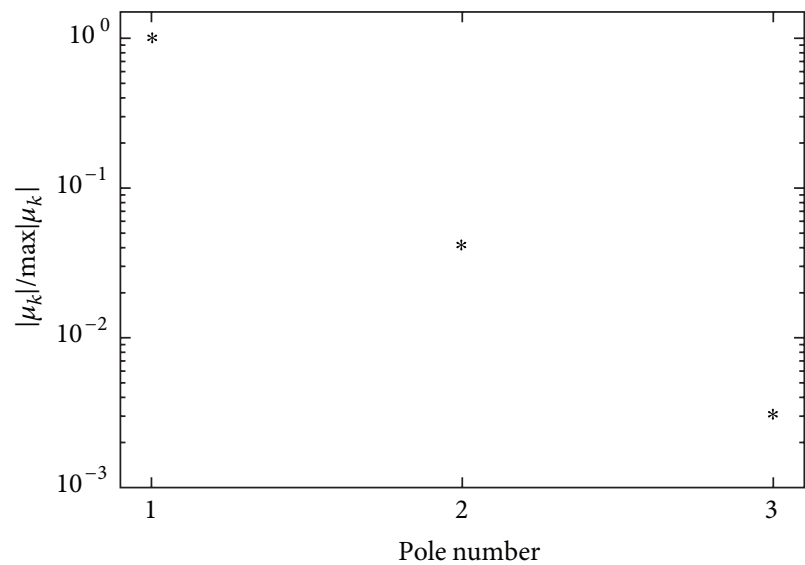

(a)

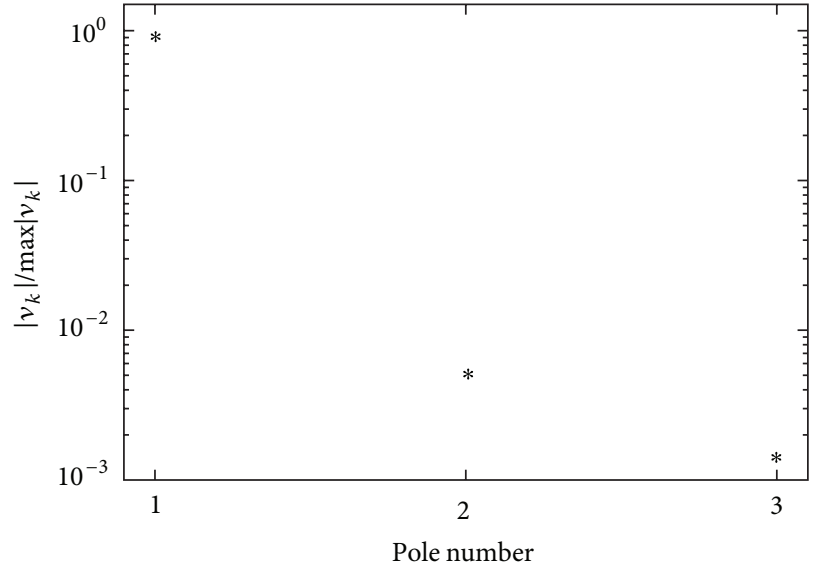

(b)

FIGURE 10: Estimation of $N$ in Case 1 using the dipole-quadrupole model (DQM) when assuming $N^{\prime}=3$. From $\left|\mu_{3} / \mu_{2}\right|=0.041$ and $\left|v_{3} / v_{2}\right|=0.004$, DQM estimates $N=1$.

the longitudinal direction, and hence a total of 30 dipoles on the half-cylinder. All the dipoles are aligned perpendicular to the surface of the cylinder to model the fact that the dipoles are perpendicular to the cerebral surface. We examined the following two cases.

Case 1. A single half-cylinder source at $\mathbf{r}_{1}=(20,50,20) \mathrm{mm}$. The vectors which determine the posture of the cylinder, $\mathbf{e}_{12}$ and $\mathbf{e}_{11}$, are set to be $\mathbf{e}_{r}$ and $\mathbf{e}_{\theta}$, respectively, where $\mathbf{e}_{r}$ and $\mathbf{e}_{\theta}$ are the unit vectors in the $r$ and $\theta$ directions at $\mathbf{r}_{1}$. (see Figure $8(\mathrm{a})$ ). In this case, the total dipole moment $\mathbf{p}_{1}$ is almost parallel to $\mathbf{r}_{1}$; the angle between them is 2.4 degrees. Since a radial dipole is a silent source for the radial component of the magnetic field [1], this cylindrical source is regarded as being almost quadrupolar.

Case 2. The same half-cylinder at $\mathbf{r}_{1}$ as in Case 1, but with $\mathbf{e}_{12}=-\mathbf{e}_{\theta}$ and $\mathbf{e}_{11}=\mathbf{e}_{r}$ (Figure 8(b)). In this case, the angle between $\mathbf{r}_{1}$ and $\mathbf{p}_{1}$ is 87 degrees, so that the source has a detectable equivalent dipole moment as well as the equivalent quadrupole moment.
We computed the forward solution generated by the 30 elemental dipoles using (1). Note that (11) was not used to compute the theoretical data. $N^{\prime}=3$ was assumed to estimate $N$.

4.5.1. Case 1. Figure 9 shows the estimated $\left|\mu_{k}\right|$ normalized by $\max \left\{\mu_{k}\right\}$ using DM. From the fact that $\left|\mu_{3} / \mu_{2}\right|=0.005$, $N$ is estimated to be two using DM. The reason why not a single equivalent dipole but two dipoles are estimated for a single cylindrical source is that the sum of the dipoles on the half-cylindrical surface is directed to the radial direction which is "silent" to the radial magnetic field outside the head. In contrast, Figures 10(a) and 10(b) show the estimated $\left|\mu_{k}\right|$ normalized by $\max \left\{\mu_{k}\right\}$ and $\left|v_{k}\right|$ normalized by $\max \left\{v_{k}\right\}$, respectively, using DQM. From $\left|\mu_{3} / \mu_{2}\right|=0.041$ and $\left|v_{3} / \nu_{2}\right|=$ $0.004, N$ is estimated to be one.

Figure 11 shows the localization result using DM and DQM. It is observed that the two positions estimated with DM were far apart from the cylindrical surface, whereas DQM well estimated the center of the cylinder. This is a great 


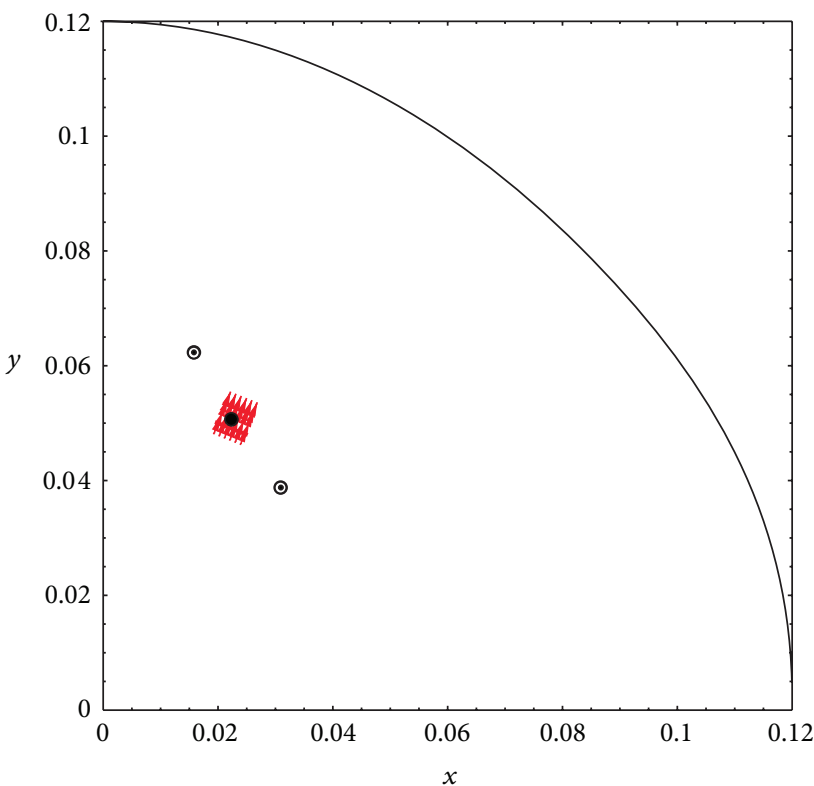

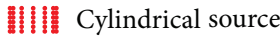

- Estimated with DQM

- Estimated with DM

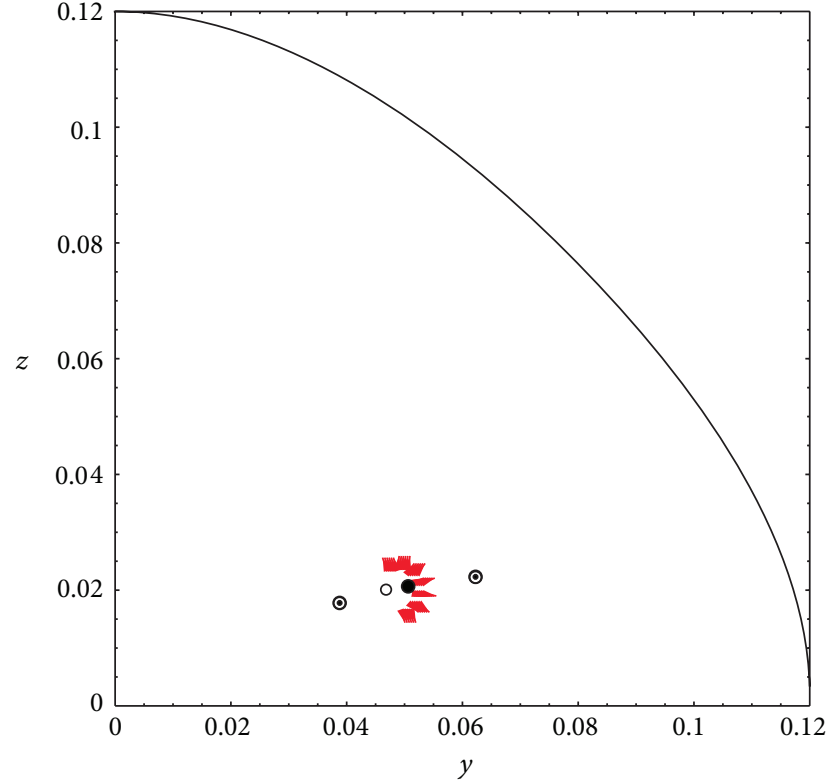

㘗潇 Cylindrical source

Estimated with DQM

- Estimated with DM

(a)

(b)

FIGURE 11: Localization result of the cylindrically distributed dipole sources using dipole model (DM) and dipole-quadrupole model (DQM).

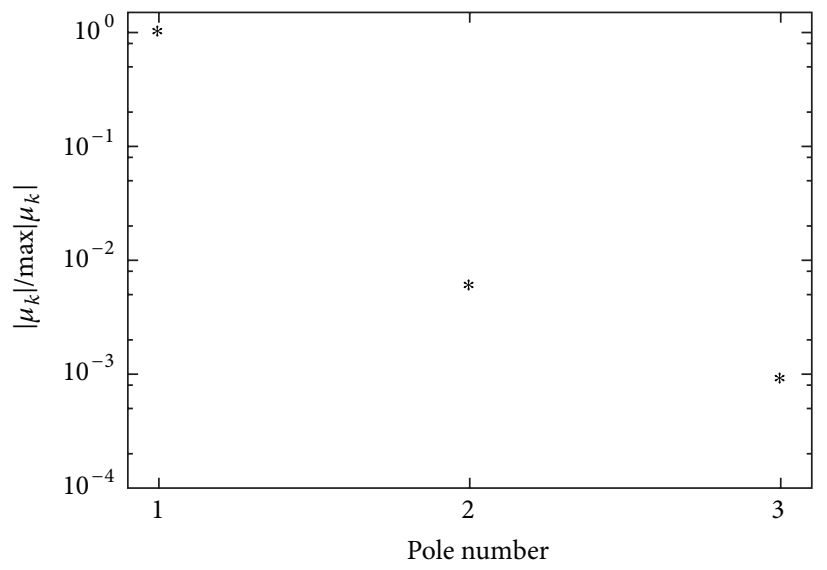

Figure 12: Estimation of $N$ in Case 1 using the dipole model (DM) when assuming $N^{\prime}=3$. From $\left|\mu_{3} / \mu_{2}\right|=0.006$, DM estimates $N=1$.

advantage of DQM; although it is difficult for DM to estimate stably two, close, oppositely directed dipoles which are tangential to the spherical surface, it is easy for DQM.

4.5.2. Case 2. Figures $12,13(a)$, and $13(\mathrm{~b})$ show the estimated $\left|\mu_{k}\right|$ using DM and $\left|\mu_{k}\right|$ and $\left|v_{k}\right|$ using DQM, respectively. In this Case, from $\left|\mu_{2} / \mu_{1}\right|=0.006$ in Figure 12 using DM and $\left|\mu_{2} / \mu_{1}\right|=0.006$ and $\left|v_{2} / v_{1}\right|=0.073$ in Figure 13 using DQM, both model estimate $N=1$. This is because the total of the elemental dipoles on the cylinder is almost perpendicular to the radial direction in Case 2 so that this cylindrical source can be regarded as a single equivalent current dipole.
Figure 14 shows the localization result. Both DM and DQM can well estimate the center position of the cylindrical source in this case.

\section{Conclusion}

We considered an inverse source problem of the Poisson equation for the radial component of the magnetic flux density when the current source is equivalently represented by multiple dipoles and quadrupoles in layered, spherical domains. By expressing the magnetic field in terms of the spherical harmonic expansion, we showed that the sectorial 


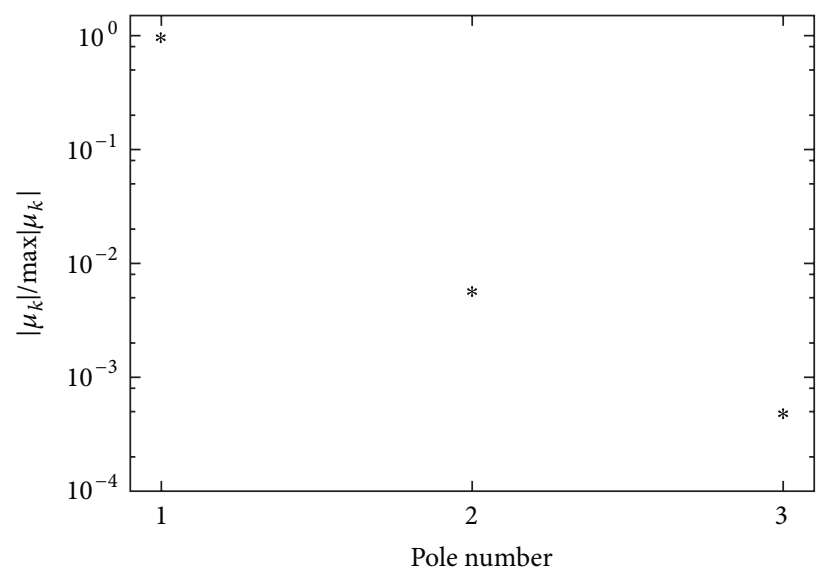

(a)

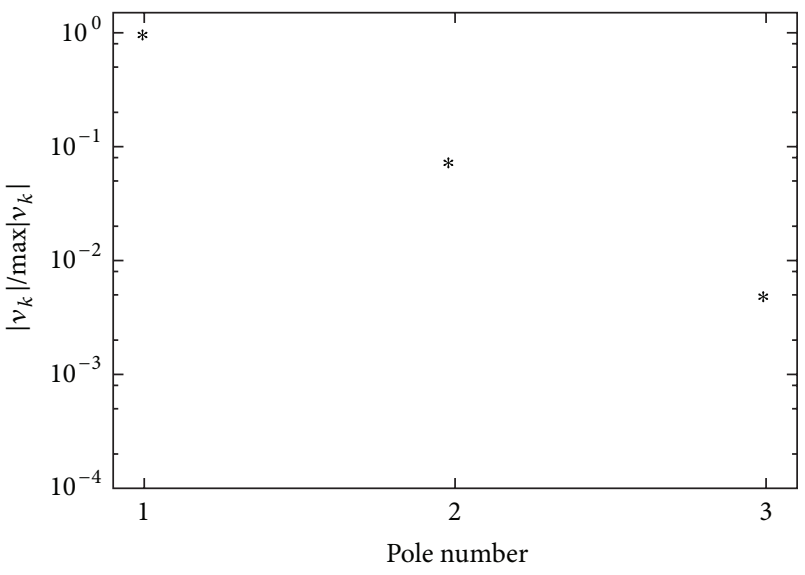

(b)

Figure 13: Estimation of $N$ in Case 1 using the dipole-quadrupole model (DQM) when assuming $N^{\prime}=3$. From $\left|\mu_{3} / \mu_{2}\right|=0.006$ and $\left|\nu_{3} / \nu_{2}\right|=$ 0.072 , DQM estimates $N=1$.

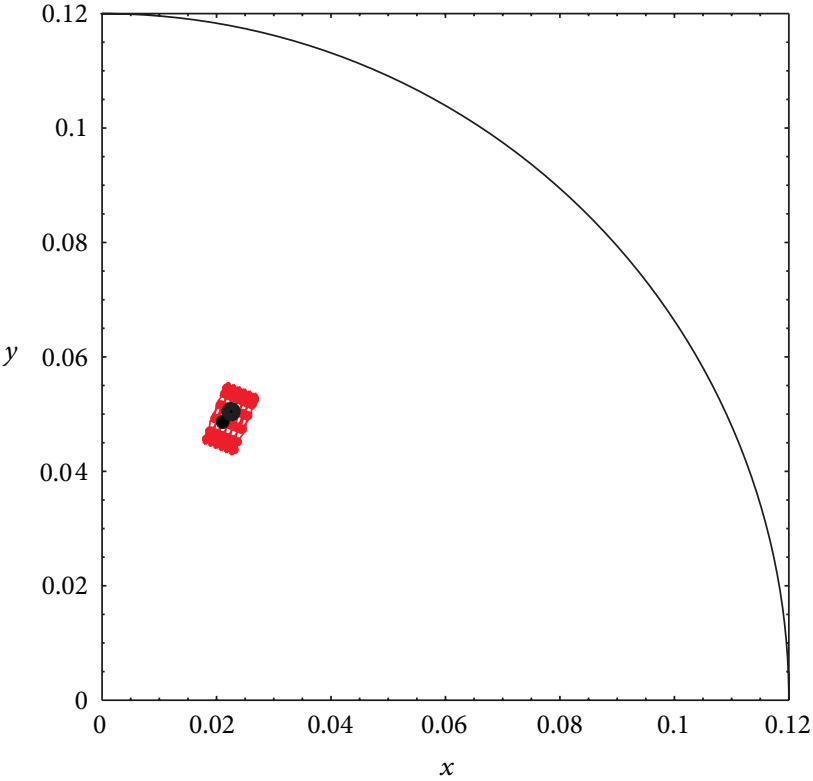

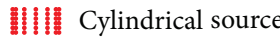

- Estimated with DQM

- Estimated with DM

(a)

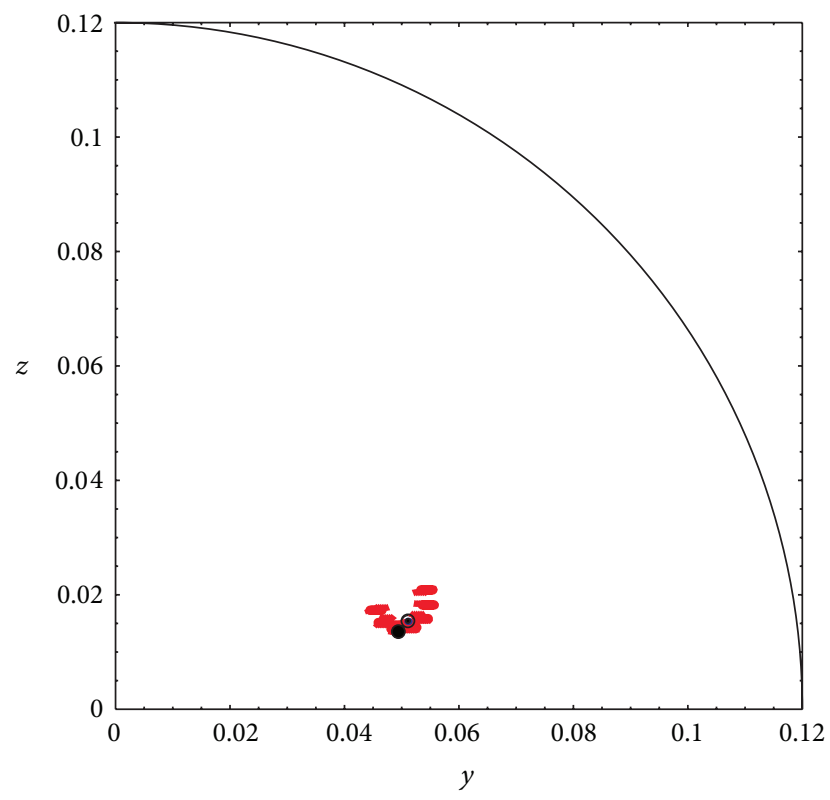

㘗雔 Cylindrical source

Estimated with DQM

- Estimated with DM

(b)

FIGURE 14: Localization result of the cylindrically distributed dipole sources using dipole model (DM) and dipole-quadrupole model (DQM).

harmonics gave the simultaneous algebraic equations relating the dipole and quadrupole parameters to the boundary data. The equations had the same form as derived in the simple- and double-pole reconstruction problem in the $2 \mathrm{D}$ case [17], so that the source parameters can be algebraically reconstructed. We proposed a method to obtain the $x$ - and $y$-coordinates of the dipole-quadrupole source by solving the simultaneous second-degree equations, with the result of which the $z$-coordinates are also determined. Numerical simulations show that the proposed algorithm estimates the number and centroid positions of the spatially extended dipoles well, especially when they include elemental dipoles oriented in opposite directions and their equivalent dipole moment is almost parallel to the radial direction of the spherical domain. A theoretical analysis to determine the thresholds depending on the noise in the algorithm for the estimation of the number of the dipole-quadrupole sources as well as an extension of the method to the case when the data is available only for part of the spherical boundary is left for further research. 


\section{Appendices}

\section{A. Derivation of (6)}

Substituting the Taylor expansion of $\nabla^{\prime}\left(1 /\left|\mathbf{r}-\mathbf{r}^{\prime}\right|\right)$ into the right-hand side of (3), we have

$$
\begin{aligned}
\mathbf{r} \cdot \mathbf{B}(\mathbf{r})= & \frac{\mu_{0}}{4 \pi} \sum_{k=1}^{N} \int_{D_{k}}\left(\left.\nabla^{\prime} \frac{1}{\left|\mathbf{r}-\mathbf{r}^{\prime}\right|}\right|_{\mathbf{r}^{\prime}=\mathbf{r}_{k}} \times \mathbf{r}\right) \cdot \mathbf{j}_{p}\left(\mathbf{r}^{\prime}\right) d v^{\prime} \\
& +\frac{\mu_{0}}{4 \pi} \sum_{k=1}^{N} \int_{D_{k}}\left(\left.\nabla^{\prime} \nabla^{\prime} \frac{1}{\left|\mathbf{r}-\mathbf{r}^{\prime}\right|}\right|_{\mathbf{r}^{\prime}=\mathbf{r}_{k}}\left(\mathbf{r}^{\prime}-\mathbf{r}_{k}\right) \times \mathbf{r}\right) \\
& \cdot \mathbf{j}_{p}\left(\mathbf{r}^{\prime}\right) d v^{\prime}+\cdots
\end{aligned}
$$

The first term of (A.1) is rewritten as

$$
\begin{aligned}
\int_{D_{k}}\left(\left.\nabla^{\prime} \frac{1}{\left|\mathbf{r}-\mathbf{r}^{\prime}\right|}\right|_{\mathbf{r}^{\prime}=\mathbf{r}_{k}} \times \mathbf{r}\right) \cdot \mathbf{j}_{p}\left(\mathbf{r}^{\prime}\right) d v^{\prime} \\
\quad=\int_{D_{k}}\left(\frac{\mathbf{r}-\mathbf{r}_{k}}{\left|\mathbf{r}-\mathbf{r}_{k}\right|^{3}} \times \mathbf{r}\right) \cdot \mathbf{j}_{p}\left(\mathbf{r}^{\prime}\right) d v^{\prime} \\
=\int_{D_{k}}\left(\frac{\mathbf{r}-\mathbf{r}_{k}}{\left|\mathbf{r}-\mathbf{r}_{k}\right|^{3}} \times \mathbf{r}_{k}\right) \cdot \mathbf{j}_{p}\left(\mathbf{r}^{\prime}\right) d v^{\prime} \\
=\left(\mathbf{r}_{k} \times \int_{D_{k}} \mathbf{j}_{p}\left(\mathbf{r}^{\prime}\right) d v^{\prime}\right) \cdot \frac{\mathbf{r}-\mathbf{r}_{k}}{\left|\mathbf{r}-\mathbf{r}_{k}\right|^{3}} .
\end{aligned}
$$

The second term of (A.1) is rewritten as

$$
\begin{aligned}
& \int_{D_{k}}\left(\left.\nabla^{\prime} \nabla^{\prime} \frac{1}{\left|\mathbf{r}-\mathbf{r}^{\prime}\right|}\right|_{\mathbf{r}^{\prime}=\mathbf{r}_{k}}\left(\mathbf{r}^{\prime}-\mathbf{r}_{k}\right) \times \mathbf{r}\right) \cdot \mathbf{j}_{p}\left(\mathbf{r}^{\prime}\right) d v^{\prime} \\
& =\int_{D_{k}}\left(\left.\nabla^{\prime} \frac{\mathbf{r}-\mathbf{r}^{\prime}}{\left|\mathbf{r}-\mathbf{r}^{\prime}\right|^{3}}\right|_{\mathbf{r}^{\prime}=\mathbf{r}_{k}}\left(\mathbf{r}^{\prime}-\mathbf{r}_{k}\right) \times \mathbf{r}\right) \cdot \mathbf{j}_{p}\left(\mathbf{r}^{\prime}\right) d v^{\prime} \\
& =\int_{D_{k}}\left(\left.\left(\nabla^{\prime} \frac{1}{\left|\mathbf{r}-\mathbf{r}^{\prime}\right|^{3}}\right)\left(\mathbf{r}-\mathbf{r}^{\prime}\right)\right|_{\mathbf{r}^{\prime}=\mathbf{r}_{k}}\left(\mathbf{r}^{\prime}-\mathbf{r}_{k}\right) \times \mathbf{r}\right) \\
& +\int_{D_{k}}\left(\left.\frac{\nabla^{\prime}\left(\mathbf{r}-\mathbf{r}^{\prime}\right)}{\left|\mathbf{r}-\mathbf{r}^{\prime}\right|^{3}}\right|_{\mathbf{r}^{\prime}=\mathbf{r}_{k}}\left(\mathbf{r}^{\prime}-\mathbf{r}_{k}\right) \times \mathbf{r}\right) \cdot \mathbf{j}_{p}\left(\mathbf{r}^{\prime}\right) d v^{\prime} \\
& =\int_{D_{k}}\left(\left.\frac{3\left(\mathbf{r}-\mathbf{r}^{\prime}\right)\left(\mathbf{r}-\mathbf{r}^{\prime}\right)}{\left|\mathbf{r}-\mathbf{r}^{\prime}\right|^{5}}\right|_{\mathbf{r}^{\prime}=\mathbf{r}_{k}}\left(\mathbf{r}^{\prime}-\mathbf{r}_{k}\right) \times \mathbf{r}\right) \cdot \mathbf{j}_{p}\left(\mathbf{r}^{\prime}\right) d v^{\prime}
\end{aligned}
$$

$$
\begin{aligned}
+\int_{D_{k}}\left(\left.\frac{-I}{\left|\mathbf{r}-\mathbf{r}^{\prime}\right|^{3}}\right|_{\mathbf{r}^{\prime}=\mathbf{r}_{k}}\left(\mathbf{r}^{\prime}-\mathbf{r}_{k}\right) \times \mathbf{r}\right) \\
\cdot \mathbf{j}_{p}\left(\mathbf{r}^{\prime}\right) d v^{\prime} \quad(I: 3 \times 3 \text { identity matrix })
\end{aligned}
$$$$
=\int_{D_{k}}\left(\frac{3\left(\mathbf{r}-\mathbf{r}_{k}\right)}{\left|\mathbf{r}-\mathbf{r}_{k}\right|^{5}}\left(\mathbf{r}-\mathbf{r}_{k}\right) \cdot\left(\mathbf{r}^{\prime}-\mathbf{r}_{k}\right) \times \mathbf{r}\right) \cdot \mathbf{j}_{p}\left(\mathbf{r}^{\prime}\right) d v^{\prime}
$$$$
-\int_{D_{k}}\left(\frac{\mathbf{r}^{\prime}-\mathbf{r}_{k}}{\left|\mathbf{r}-\mathbf{r}_{k}\right|^{3}} \times \mathbf{r}\right) \cdot \mathbf{j}_{p}\left(\mathbf{r}^{\prime}\right) d v^{\prime}
$$$$
=\int_{D_{k}}\left(\frac{3\left(\mathbf{r} \times \mathbf{r}_{k}\right)\left(\mathbf{r}-\mathbf{r}_{k}\right)}{\left|\mathbf{r}-\mathbf{r}_{k}\right|^{5}}\left(\mathbf{r}^{\prime}-\mathbf{r}_{k}\right)\right) \cdot \mathbf{j}_{p}\left(\mathbf{r}^{\prime}\right) d v^{\prime}
$$$$
+\int_{D_{k}} X_{r} \frac{\mathbf{r}^{\prime}-\mathbf{r}_{k}}{\left|\mathbf{r}-\mathbf{r}_{k}\right|^{3}} \cdot \mathbf{j}_{p}\left(\mathbf{r}^{\prime}\right) d v^{\prime} .
$$

Here, for a $3 \times 3$ matrix $A$ whose row vectors are $\mathbf{a}_{1}^{T}, \mathbf{a}_{2}^{T}$, and $\mathbf{a}_{3}^{T}$, it holds that

$$
(A \mathbf{x}) \cdot \mathbf{y}=\left(\begin{array}{c}
\mathbf{a}_{1}^{T} \mathbf{x} \\
\mathbf{a}_{2}^{T} \mathbf{x} \\
\mathbf{a}_{3}^{T} \mathbf{x}
\end{array}\right) \cdot \mathbf{y}=\mathbf{a}_{1}^{T} \mathbf{x} y_{1}+\mathbf{a}_{2}^{T} \mathbf{x} y_{2}+\mathbf{a}_{3}^{T} \mathbf{x} y_{3}=A: \mathbf{y} \mathbf{x}
$$

Using this property of the tensor contraction, (A.3) is further rewritten as

$$
\begin{aligned}
\frac{3\left(\mathbf{r} \times \mathbf{r}_{k}\right)\left(\mathbf{r}-\mathbf{r}_{k}\right)}{\left|\mathbf{r}-\mathbf{r}_{k}\right|^{5}} & : \int_{D_{k}} \mathbf{j}_{p}\left(\mathbf{r}^{\prime}\right)\left(\mathbf{r}^{\prime}-\mathbf{r}_{k}\right) d v^{\prime}+\frac{X_{r}}{\left|\mathbf{r}-\mathbf{r}_{k}\right|^{3}} \\
& : \int_{D_{k}} \mathbf{j}_{p}\left(\mathbf{r}^{\prime}\right)\left(\mathbf{r}^{\prime}-\mathbf{r}_{k}\right) d v^{\prime} \\
& =\frac{3\left(\mathbf{r} \times \mathbf{r}_{k}\right)\left(\mathbf{r}-\mathbf{r}_{k}\right)+\left|\mathbf{r}-\mathbf{r}_{k}\right|^{2} X_{r}}{\left|\mathbf{r}-\mathbf{r}_{k}\right|^{5}} \\
& : \int_{D_{k}} \mathbf{j}_{p}\left(\mathbf{r}^{\prime}\right)\left(\mathbf{r}^{\prime}-\mathbf{r}_{k}\right) d v^{\prime}
\end{aligned}
$$

Therefore, substituting (A.2) and (A.5) into (3) gives (6).

B.

This can be shown as follows. It is easy to obtain the dipole terms. For the quadrupole terms, we use the identity:

$$
\int_{\Omega_{1}} \mathbf{a}\left(\mathbf{r}^{\prime}\right) \cdot Q_{k} \nabla^{\prime} \delta\left(\mathbf{r}^{\prime}-\mathbf{r}_{k}\right) d v^{\prime}=Q:\left.\left(\nabla^{\prime} \mathbf{a}\left(\mathbf{r}^{\prime}\right)\right)^{T}\right|_{\mathbf{r}^{\prime}=\mathbf{r}_{k}}
$$

for an arbitrary vector field $\mathbf{a}\left(\mathbf{r}^{\prime}\right)=\left(a_{x}\left(\mathbf{r}^{\prime}\right), a_{y}\left(\mathbf{r}^{\prime}\right), a_{z}\left(\mathbf{r}^{\prime}\right)\right)^{T}$, where $T$ represents the transpose. When inserting 
the quadrupole terms in (12) into (1), we have from (B.1)

$$
\begin{array}{r}
\int_{\Omega_{1}}\left(\nabla^{\prime} \frac{1}{\left|\mathbf{r}-\mathbf{r}^{\prime}\right|} \times \mathbf{r}^{\prime}\right) \cdot Q_{k} \nabla^{\prime} \delta\left(\mathbf{r}^{\prime}-\mathbf{r}_{k}\right) d v^{\prime} \\
=Q_{k}:\left.\left(\nabla^{\prime}\left(\nabla^{\prime} \frac{1}{\left|\mathbf{r}-\mathbf{r}^{\prime}\right|} \times \mathbf{r}^{\prime}\right)\right)^{T}\right|_{\mathbf{r}^{\prime}=\mathbf{r}_{k}} .
\end{array}
$$

Here, it holds that

$$
\begin{aligned}
& \nabla^{\prime}\left(\nabla^{\prime} \frac{1}{\left|\mathbf{r}-\mathbf{r}^{\prime}\right|} \times \mathbf{r}^{\prime}\right) \\
& \quad=\nabla^{\prime} \frac{\mathbf{r} \times \mathbf{r}^{\prime}}{\left|\mathbf{r}-\mathbf{r}^{\prime}\right|^{3}}=\left(\nabla^{\prime} \frac{1}{\left|\mathbf{r}-\mathbf{r}^{\prime}\right|^{3}}\right)\left(\mathbf{r} \times \mathbf{r}^{\prime}\right)+\frac{\nabla^{\prime}\left(\mathbf{r} \times \mathbf{r}^{\prime}\right)}{\left|\mathbf{r}-\mathbf{r}^{\prime}\right|^{3}} \\
& =\frac{3\left(\mathbf{r}-\mathbf{r}^{\prime}\right)\left(\mathbf{r} \times \mathbf{r}^{\prime}\right)}{\left|\mathbf{r}-\mathbf{r}^{\prime}\right|^{5}}+\frac{1}{\left|\mathbf{r}-\mathbf{r}^{\prime}\right|^{3}}\left(\begin{array}{ccc}
0 & z & -y \\
-z & 0 & x \\
y & -x & 0
\end{array}\right),
\end{aligned}
$$

and hence

$$
\begin{aligned}
& \left.\left(\nabla^{\prime}\left(\nabla^{\prime} \frac{1}{\left|\mathbf{r}-\mathbf{r}^{\prime}\right|} \times \mathbf{r}^{\prime}\right)\right)^{T}\right|_{\mathbf{r}^{\prime}=\mathbf{r}_{k}} \\
& \quad=\frac{3\left(\mathbf{r} \times \mathbf{r}_{k}\right)\left(\mathbf{r}-\mathbf{r}_{k}\right)}{\left|\mathbf{r}-\mathbf{r}_{k}\right|^{5}}+\frac{X_{r}}{\left|\mathbf{r}-\mathbf{r}_{k}\right|^{3}} .
\end{aligned}
$$

Thus we have (11).

\section{Derivation of (21)}

It holds that

$$
\begin{aligned}
& \mathrm{Q}_{k}:\left.\left(\nabla\left(\nabla(x+i y)^{n} \times \mathbf{r}\right)\right)^{T}\right|_{\mathbf{r}=\mathbf{r}_{k}} \\
& =\left(\begin{array}{lll}
q_{x x, k} & q_{x y, k} & q_{x z, k} \\
q_{x y, k} & q_{y y, k} & q_{y z, k} \\
q_{x z, k} & q_{y z, k} & q_{z z, k}
\end{array}\right) \\
& :\left.n\left(\begin{array}{ccc}
i(n-1) z(x+i y)^{n-2} & -(n-1) z(x+i y)^{n-2} & i(x+i y)^{n-1} \\
-(n-1) z(x+i y)^{n-2} & -i(n-1) z(x+i y)^{n-2} & -(x+i y)^{n-1} \\
-i n(x+i y)^{n-1} & n(x+i y)^{n-1} & 0
\end{array}\right)\right|_{\mathbf{r}=\mathbf{r}_{k}} \\
& =n\left(i(n-1) q_{x x, k} z_{k}\left(x_{k}+i y_{k}\right)^{n-2}-(n-1) q_{x y, k} z_{k}\left(x_{k}+i y_{k}\right)^{n-2}\right. \\
& +i q_{x z, k}\left(x_{k}+i y_{k}\right)^{n-1}-(n-1) q_{x y, k} z_{k}\left(x_{k}+i y_{k}\right)^{n-2} \\
& -i(n-1) q_{y y, k} z_{k}\left(x_{k}+i y_{k}\right)^{n-2}-q_{y z, k}\left(x_{k}+i y_{k}\right)^{n-1} \\
& \left.-i n q_{x z, k}\left(x_{k}+i y_{k}\right)^{n-1}+n q_{y z, k}\left(x_{k}+i y_{k}\right)^{n-1}\right) \\
& =n(n-1)\left(\left(i\left(q_{x x, k}-q_{y y, k}\right)-2 q_{x y, k}\right) z_{k}\right. \\
& \left.+\left(q_{y z, k}-i q_{x z, k}\right)\left(x_{k}+i y_{k}\right)\right)\left(x_{k}+i y_{k}\right)^{n-2},
\end{aligned}
$$

which gives (21).

\section{Derivation of (42) and (43)}

It is easy to see that

$$
\begin{aligned}
& \left.\nabla\left(\nabla \phi_{j}(x+i y) \times \mathbf{r}\right)\right|_{\mathbf{r}=\mathbf{r}_{k}} \\
& =\left.\left(\begin{array}{ccc}
i z \phi_{j}^{\prime \prime} & -z \phi_{j}^{\prime \prime} & -i(x+i y) \phi_{j}^{\prime \prime}-i \phi_{j}^{\prime} \\
-z \phi_{j}^{\prime \prime} & -i z \phi_{j}^{\prime \prime} & (x+i y) \phi_{j}^{\prime \prime}+\phi_{j}^{\prime} \\
i \phi_{j}^{\prime} & -\phi_{j}^{\prime} & 0
\end{array}\right)\right|_{\mathbf{r}=\mathbf{r}_{k}},
\end{aligned}
$$

where $\phi_{j}, \phi_{j}^{\prime}$, and $\phi_{j}^{\prime \prime}$ represent $\phi_{j}(x+i y), \phi_{j}^{\prime}(x+i y)$, and $\phi_{j}^{\prime \prime}(x+$ $i y)$, respectively. From the definition of $\phi_{j}$ in (37), one easily finds that $\phi_{j}\left(\zeta_{k}\right)=\phi_{j}^{\prime}\left(\zeta_{k}\right)=0$ for all $k=1,2, \ldots, N$, and hence

$$
\begin{aligned}
& \left.\nabla\left(\nabla \phi_{j}(x+i y) \times \mathbf{r}\right)\right|_{\mathbf{r}=\mathbf{r}_{k}} \\
& \quad=\left(\begin{array}{ccc}
i z_{k} \phi_{j}^{\prime \prime}\left(\zeta_{k}\right) & -z_{k} \phi_{j}^{\prime \prime}\left(\zeta_{k}\right) & -i \zeta_{k} \phi_{j}^{\prime \prime}\left(\zeta_{k}\right) \\
-z_{k} \phi_{j}^{\prime \prime}\left(\zeta_{k}\right) & -i z_{k} \phi_{j}^{\prime \prime}\left(\zeta_{k}\right) & \zeta_{k} \phi_{j}^{\prime \prime}\left(\zeta_{k}\right) \\
0 & 0 & 0
\end{array}\right) .
\end{aligned}
$$

Also, from the definition of $\phi_{j}$, we have

$$
\phi_{j}^{\prime \prime}\left(\zeta_{k}\right)=2 \delta_{j k} \prod_{l=1, l \neq j}^{N}\left(\zeta_{j}-\zeta_{l}\right)^{3},
$$

where $\delta_{j k}$ is Kronecker's delta, which gives (42). In the same way,

$$
\begin{aligned}
& \left.\nabla\left(\nabla \phi_{j}(x+i y) z \times \mathbf{r}\right)\right|_{\mathbf{r}=\mathbf{r}_{k}} \\
& =\left.\left(\begin{array}{ccc}
-y \phi_{j}^{\prime}+i z^{2} \phi_{j}^{\prime \prime} & \phi_{j}+x \phi_{j}^{\prime}-z^{2} \phi_{j}^{\prime \prime} & -i(x+i y) z \phi_{j}^{\prime \prime}-i z \phi_{j}^{\prime} \\
-\phi_{j}-i y \phi_{j}^{\prime}-z^{2} \phi_{j}^{\prime \prime} & i x \phi_{j}^{\prime}-i z^{2} \phi_{j}^{\prime \prime} & (x+i y) z \phi_{j}^{\prime \prime}+z \phi_{j}^{\prime} \\
2 i z \phi_{j}^{\prime} & -2 z \phi_{j}^{\prime} & -i(x+i y) \phi_{j}^{\prime}
\end{array}\right)\right|_{\mathbf{r}=\mathbf{r}_{k}} \\
& =z_{k}\left(\begin{array}{ccc}
i z_{k} \phi_{j}^{\prime \prime}\left(\zeta_{k}\right) & -z_{k} \phi_{j}^{\prime \prime}\left(\zeta_{k}\right) & -i \zeta_{k} \phi_{j}^{\prime \prime}\left(\zeta_{k}\right) \\
-z_{k} \phi_{j}^{\prime \prime}\left(\zeta_{k}\right) & -i z_{k} \phi_{j}^{\prime \prime}\left(\zeta_{k}\right) & \zeta_{k} \phi_{j}^{\prime \prime}\left(\zeta_{k}\right) \\
0 & 0 & 0
\end{array}\right),
\end{aligned}
$$

which gives (43).

\section{Acknowledgments}

This research has been partially supported by the Moritani Scholarship Foundation and the 2012 Inamori Research Grants Program. 


\section{References}

[1] J. Sarvas, "Basic mathematical and electromagnetic concepts of the biomagnetic inverse problem," Physics in Medicine and Biology, vol. 32, no. 1, article 4, pp. 11-22, 1987.

[2] S. Baillet, J. C. Mosher, and R. M. Leahy, "Electromagnetic brain mapping," IEEE Signal Processing Magazine, vol. 18, no. 6, pp. 14-30, 2001.

[3] G. Dassios and D. Hadjiloizi, "On the non-uniqueness of the inverse problem associated with electroencephalography," Inverse Problems, vol. 25, no. 11, Article ID 115012, 18 pages, 2009.

[4] A. S. Fokas, Y. Kurylev, and V. Marinakis, "The unique determination of neuronal currents in the brain via magnetoencephalography," Inverse Problems, vol. 20, no. 4, pp. 1067-1082, 2004.

[5] M. Huang, C. J. Aine, S. Supek, E. Best, D. Ranken, and E. R. Flynn, "Multi-start downhill simplex method for spatiotemporal source localization in magnetoencephalography," Electroencephalography and Clinical Neurophysiology, vol. 108, no. 1, pp. 32-44, 1998.

[6] T. Ohe and K. Ohnaka, "A precise estimation method for locations in an inverse logarithmic potential problem for point mass models," Applied Mathematical Modelling, vol. 18, no. 8, pp. 446-452, 1994.

[7] A. El Badia and T. Ha-Duong, "An inverse source problem in potential analysis," Inverse Problems, vol. 16, no. 3, pp. 651-663, 2000.

[8] K. Yamatani, T. Ohe, and K. Ohnaka, "An identification method of electric current dipoles in spherically symmetric conductor," Journal of Computational and Applied Mathematics, vol. 143, no. 2, pp. 189-200, 2002.

[9] T. Nara and S. Ando, "A projective method for an inverse source problem of the Poisson equation," Inverse Problems, vol. 19, no. 2, pp. 355-369, 2003.

[10] T. Nara, J. Oohama, M. Hashimoto, T. Takeda, and S. Ando, "Direct reconstruction algorithm of current dipoles for vector magnetoencephalography and electroencephalography," Physics in Medicine and Biology, vol. 52, no. 13, article 14, pp. 3859-3879, 2007.

[11] F. Darvas, D. Pantazis, E. Kucukaltun-Yildirim, and R. M. Leahy, "Mapping human brain function with MEG and EEG: methods and validation," NeuroImage, vol. 23, no. 1, pp. S289-S299, 2004.

[12] K. Matsuura and Y. Okabe, "Selective minimum-norm solution of the biomagnetic inverse problem," IEEE Transactions on Biomedical Engineering, vol. 42, no. 6, pp. 608-615, 1995.

[13] G. Nolte and G. Curio, "On the calculation of magnetic fields based on multipole modeling of focal biological current sources," Biophysical Journal, vol. 73, no. 3, pp. 1253-1262, 1997.

[14] G. Nolte and G. Curio, "Current multipole expansion to estimate lateral extent of neuronal activity: a theoretical analysis," IEEE Transactions on Biomedical Engineering, vol. 47, no. 10, pp. 1347-1355, 2000.

[15] K. Jerbi, J. C. Mosher, S. Baillet, and R. M. Leahy, "On MEG forward modelling using multipolar expansions," Physics in Medicine and Biology, vol. 47, no. 4, pp. 523-555, 2002.

[16] K. Jerbi, S. Baillet, J. C. Mosher, G. Nolte, L. Garnero, and R. M. Leahy, "Localization of realistic cortical activity in MEG using current multipoles," NeuroImage, vol. 22, no. 2, pp. 779-793, 2004.

[17] T. Nara, "An algebraic method for identification of dipoles and quadrupoles," Inverse Problems, vol. 24, no. 2, Article ID 025010, 19 pages, 2008.
[18] A. Irimia, K. R. Swinney, and J. P. Wikswo, "Partial independence of bioelectric and biomagnetic fields and its implications for encephalography and cardiography," Physical Review E, vol. 79, no. 5, Article ID 051908, 2009.

[19] T. Nara, "Reconstruction of the number and positions of dipoles and quadrupoles using an algebraic method," Journal of Physics: Conference Series, vol. 135, no. 1, Article ID 012076, 2008.

[20] T. Nara, "An explicit method for inverse reconstruction of equivalent current dipoles and quadrupoles," in Magnetoencephalography, E. W. Pang, Ed., Chapter 6, pp. 113-128, InTech, 2011.

[21] R. E. Alvarez, "Filter functions for computing multipole moments from the magnetic field normal to a plane," IEEE Transactions on Medical Imaging, vol. 10, no. 3, pp. 375-381, 1991.

[22] S. Taulu, J. Simola, and M. Kajola, "Applications of the signal space separation method," IEEE Transactions on Signal Processing, vol. 53, no. 9, pp. 3359-3372, 2005.

[23] E. W. Hobson, The Theory of Spherical and Ellipsoidal Harmonics, Cambridge University Press, 1931.

[24] P. Barone and R. March, "Some properties of the asymptotic location of poles of Padé approximants to noisy rational functions, relevant for modal analysis," IEEE Transactions on Signal Processing, vol. 46, no. 9, pp. 2448-2457, 1998.

[25] E. B. Saff and A. B. J. Kuijlaars, "Distributing many points on a sphere," The Mathematical Intelligencer, vol. 19, no. 1, pp. 5-11, 1997.

[26] T. Nara and S. Ando, "Direct localization of poles of a meromorphic function from measurements on an incomplete boundary," Inverse Problems, vol. 26, no. 1, Article ID 015011, 2010. 


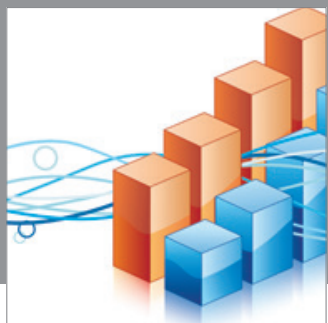

Advances in

Operations Research

mansans

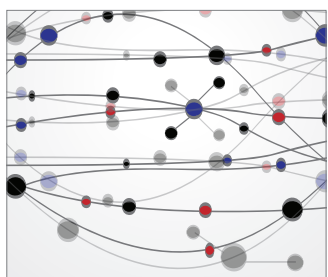

The Scientific World Journal
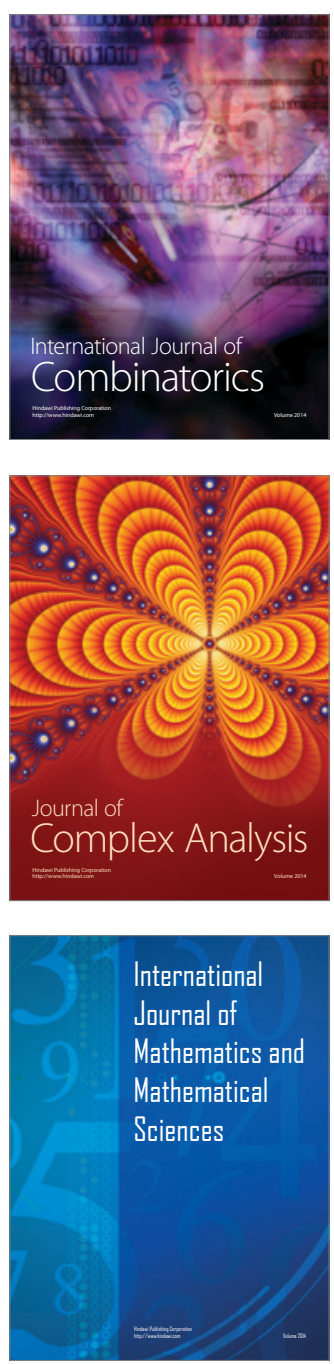
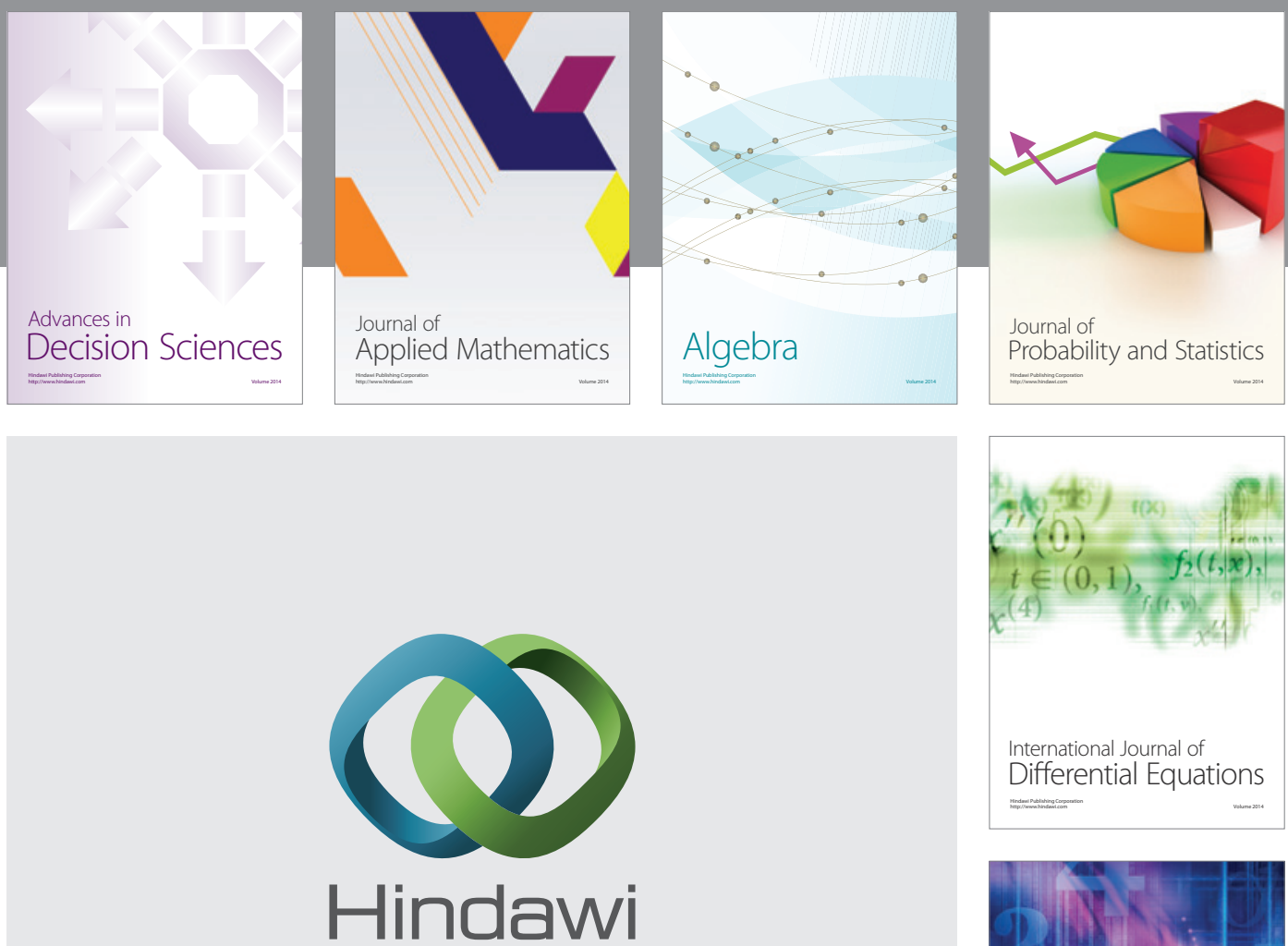

Submit your manuscripts at http://www.hindawi.com
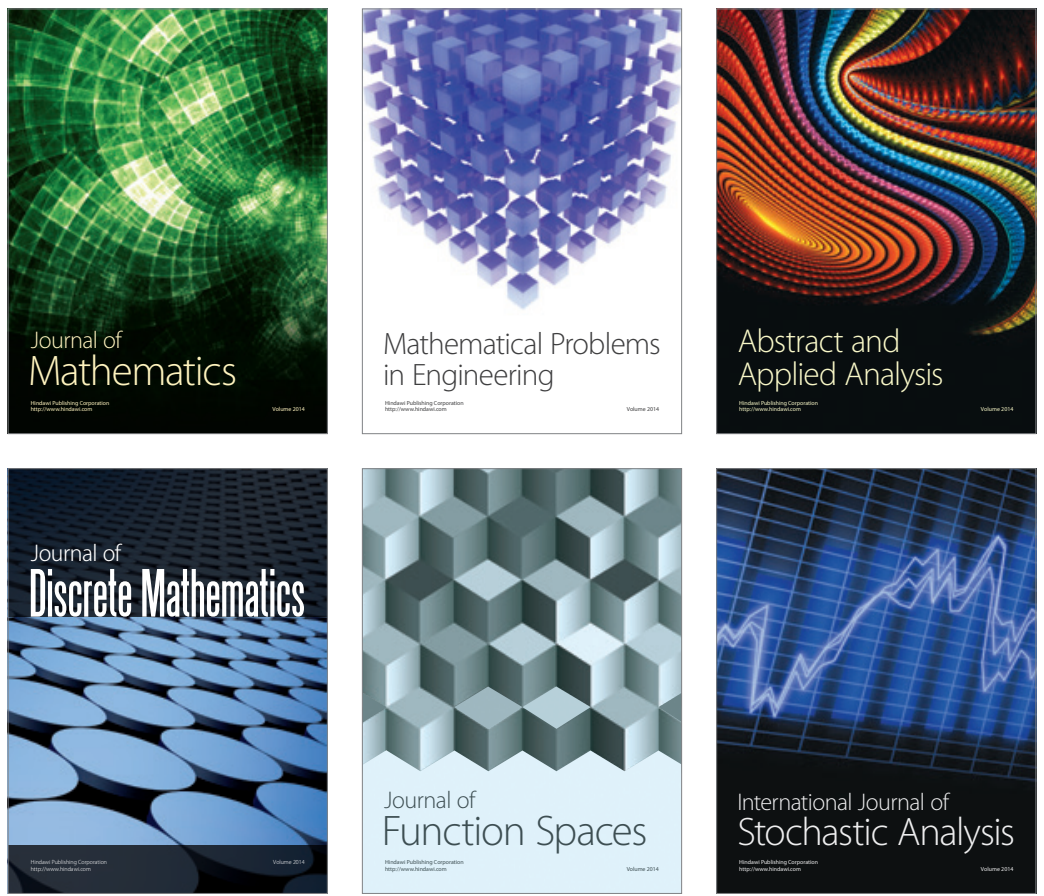

Journal of

Function Spaces

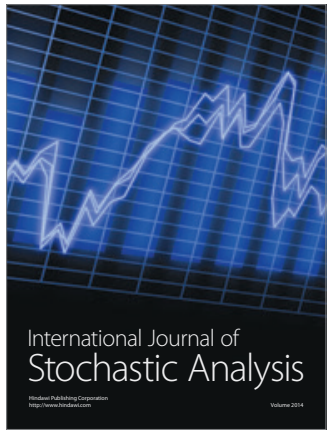

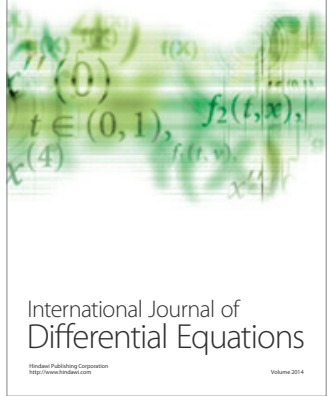
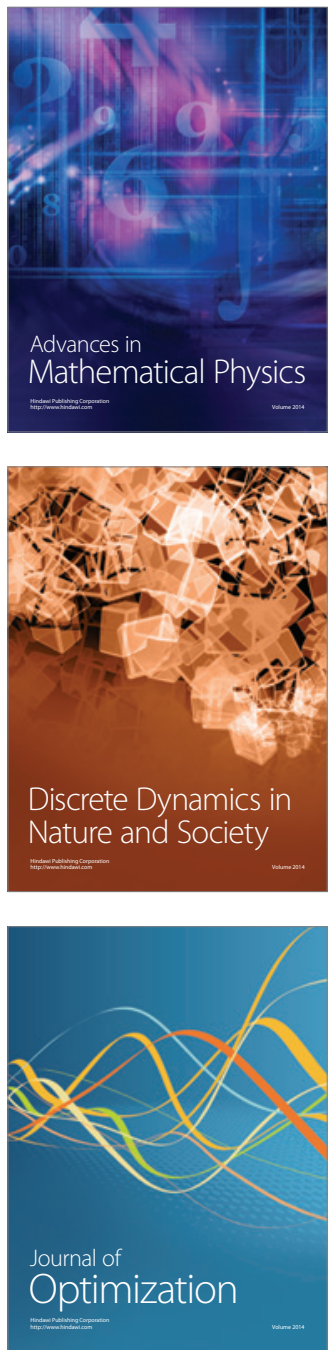